Research Article

\title{
Profitability vs. Stability: The Choice of Trading Strategies in a Capacity Sharing Supply Chain
}

\author{
Hongshuai Han (D) ${ }^{1}$ and Mengdi Yao iD $^{2}$ \\ ${ }^{1}$ College of Management, Qilu University of Technology (Shandong Academy of Sciences), Jinan 250353, China \\ ${ }^{2}$ School of Literature, Law and Economics, Wuhan University of Science and Technology, Wuhan 430065, China \\ Correspondence should be addressed to Mengdi Yao; yaomengdi@wust.edu.cn
}

Received 2 August 2021; Accepted 9 November 2021; Published 13 December 2021

Academic Editor: Baogui Xin

Copyright (C) 2021 Hongshuai Han and Mengdi Yao. This is an open access article distributed under the Creative Commons Attribution License, which permits unrestricted use, distribution, and reproduction in any medium, provided the original work is properly cited.

\begin{abstract}
Supported by a third-party capacity sharing platform, manufacturers can share capacities with others to match the rapidly changing demand. Both the capacity requestor and the capacity provider can choose to seek or wait for matches, forming different trading strategies (capacity- and demand-driven strategies). Based on the game and chaos theories, this paper analyzes the preference of the capacity provider, the capacity requestor, and the capacity platform operator on different trading strategies from the aspects of profitability and stability. It finds that the platform operator values stability much more than profitability, although the latter may reach higher optimal expected profits. The preference of each supply chain member is influenced by the production cost, potential market size, and the limited capacity of the capacity requestor. A stable system can result in higher long-run profits than a profitable system. We further propose the all-win situation for the capacity provider, capacity requestor, and platform operator.
\end{abstract}

\section{Introduction}

Manufacturing capacity sharing can efficiently solve the problem of mismatch between demand and supply. Supported by a third-party capacity-sharing platform, the capacity requestor can more easily find the appropriate capacity provider with available surplus capacity. The reason is that the third-party capacity-sharing platform links dispersed manufacturers and offers an effective manufacturing solution to facilitate the match between capacity supply and demand. On the manufacturing capacity sharing platform, both the capacity requestor and the capacity provider can choose either to actively seek a well-matched partner or to wait for the inquiry, considering the trade-off between cost and lead time.

If the capacity requestor acts as a waiter, it can announce a request for quotation (RFQ) and release the information about quotation duration, product category, processing location, processing quantity, and delivery time, waiting for the capacity provider's quotation. Under this mechanism, it can present the range of the acceptable price but lose the first-mover advantage of pricing. Meanwhile, it may obtain low-cost service but face a long production lead time. On the contrary, if it chooses to be a seeker, it can keep searching based on its specific needs until it finds a satisfactory wellmatched partner. Under this mechanism, it can decide on the quantity and then affect the capacity-transfer price. The demand can be met at a relatively rapid speed, while the corresponding cost may be high. Similarly, when the capacity provider chooses to be a waiter, it releases the information about its capacity and processing schedule and waits to be selected. It can also seek capacity requestors based on their RFQ so that it can hold the first-mover advantage of pricing. In addition to the capacity requestor and the capacity provider, the platform operator as the rule maker can facilitate the capacity-sharing transaction by offering recommendation services.

The first-mover advantage also has great impact on the profitability of the firm. Waiting for partners, the firm can save information search costs but lose first-mover decision- 
making advantage to some extent. It may even put itself in a fiercely competitive market. On the contrary, it is reasonable that the firm actively seeks matches. Considering both the capacity requestor and the capacity provider can choose to be a waiter or a seeker, we consider two trading strategies, that is, the demand- and the capacity-driven strategies. For example, Figure 1 shows two trading strategies in the INDICS international cloud platform (http://www.indics.com/ ). Under the demand-driven strategy (DD), the capacity requestor releases demand information and waits for the capacity providers' quotations. Under the capacity-driven strategy (CD), the capacity provider releases capacity information and waits for the capacity requestor's inquiry. The choice of trading strategies is influenced by many factors, such as the urgency of demand, the degree of capacity limitation, and the production cost.

While most of the existing literature focuses on profitability, this paper emphasizes the importance of the stability of the decision-making system. Stability and profitability are interacted $[1,2]$. Only in a stable system can a firm obtain optimal decisions and expected profits. In the actual case, decision-makers cannot obtain perfect information about the market. For instance, it is difficult for the operator of the CASICloud platform to gain all information on participants and be completely rational. It may be affected by heterogeneous behavioral factors when making decisions. These bounded rational decision-makers have to adjust their decisions according to the marginal profits in each period. Therefore, the decision-making process can be modeled as a nonlinear difference equation. Based on chaos theory, the equilibrium points may be unstable as system parameters change. That is to say, the decision-maker may not get the optimal solutions, or the optimal solutions cannot bear the change of environment. In such an unstable system, the bifurcation and chaos phenomenon may occur. As a result, the decision-maker should pay more attention to stability and pursue the balance between profitability and stability.

In this paper, we focus on the decision-making process in a capacity sharing supply chain, investigating which trading strategy is preferable for the platform operator, the capacity requestor, and the capacity provider, respectively. Specifically, this paper explores the following problems:

(1) Which trading strategy is more profitable for a perfect rational platform operator?

(2) What is the condition for the stability of the equilibrium outcomes?

(3) Which trading strategy is more favorable for a bounded rational platform operator?

(4) How does stability influence the accumulated profit of the system?

(5) How to select the trading strategy for each participant in the capacity sharing?

The academic contributions of this research are threefold. First, this paper investigates the profitability performance and the stability performance of the capacity- and demand-driven trading strategies in the manufacturing capacity sharing platform. Second, this paper explores the preference of platform operators on trading strategy under both perfect rationality and bounded rationality. Third, this paper analyzes the trading strategy selection of both the capacity provider and the capacity requestor and further shows the all-win situation.

The remainder of this paper is organized as follows. A literature review is presented in Section 2. Section 3 describes the basic models and assumptions. We perform the main analysis for the two market structures in Section 4 and make a comparison in Section 5. The study is extended for all-win situation analysis in Section 6. Section 7 concludes this study. All expressions and proofs are provided in Appendix A and Appendix B.

\section{Literature Review}

This paper studies the trade-off between profitability and stability and the choice of trading strategies in a capacitysharing supply chain. In this paper, stability is measured based on the chaos theory. Therefore, this paper is related to two research streams in the literature: the manufacturing capacity sharing and the application of chaos theory in the supply chain.

2.1. Manufacturing Capacity Sharing. As an effective way to solve the capacity limitation problem, capacity sharing has attracted more and more attention from both academia and practitioners in recent years. In the manufacturing industry, Guo and $\mathrm{Wu}$ [3] investigated optimal strategies and firm profitability of capacity sharing between competing firms under both ex ante and ex post contracting. Renna and Argoneto [4] propose a cooperative approach based on game theory for a network of independent factories facing the capacity-sharing issue. Considering that each firm has an existing capacity and faces both fixed and variable costs in purchasing additional capacity, Yang and Anderson [5] compare the outcomes obtained in the scenarios where the firms simultaneously (or sequentially) make their expansion decisions and then simultaneously decide their production decisions. Seok and Nof [6] propose the capacity sharing model among independent and noncompetitive manufacturers. Wang et al. [7] built a resource allocation model considering the credit of resource providers and adopted a case study of validating the model's effectiveness. Yang et al. [8] propose a bipartite matching framework to study the resource allocation among customers and manufacturers in additive manufacturing under sharing economy. Li and Jiang [9] investigate the definition, components, operational logic, and classification of the enhanced self-organizing agent in the context of the sustainable sharing factory to enhance the utilization of the shared manufacturing resources.

With the rapid development of the Internet, third-party platform operator helps eliminate barriers to transactions, making more and more small- and medium-sized 


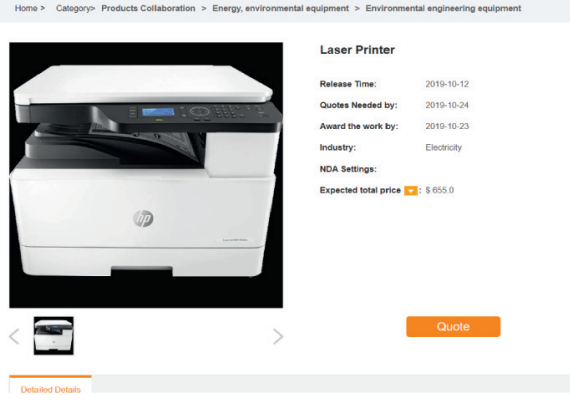

(a)

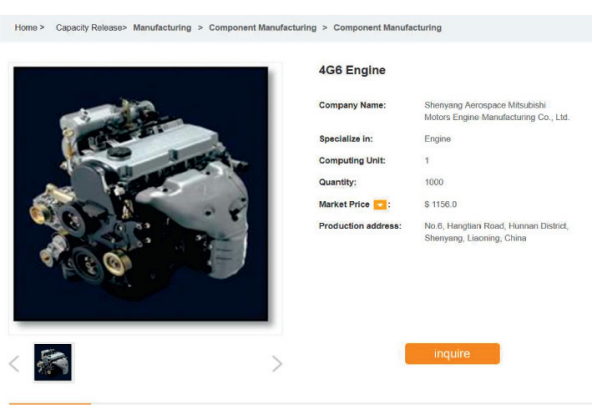

(b)

FIgURE 1: The cases of two trading strategies: (a) demand-driven case and (b) capacity-driven case.

enterprises (SMEs) join the capacity-sharing business. Qin et al. [10] studied two competing manufacturers shared capacities through a platform. With a revenue-sharing contract, the profit of the firm with insufficient capacity increased. Considering high- and low-value matchers, Basu et al. [11] examined how the matchers should select each other and how the platform should price its search and authentication services. Zhao et al. [12] study the decision and coordination for a capacity-sharing supply chain when the platform charge fixed and quality-based transaction fee. Ye et al. [13] investigate the competition between a manufacturer and a sharing economy platform considering the strategic consumers and sharing utility brought by the sharing transaction. Szaller et al. [14] introduce a novel mutualistic framework to support the strategic collaboration of manufacturing facilities and enhance resource utilization. Li et al. [15] propose a blockchain-based digital twin sharing platform to protect the copyright and knowledge of a digital twin in the sharing process to facilitate the manufacturing resource integration.

To the best of our knowledge, very few literature focuses on trading strategy choices in a capacity sharing supply chain. We study how the platform operator sets a trading strategy for the manufacturers making different products with different production costs considering the profitability and stability of the strategies and how the decisions affect the choices of the manufacturers.

2.2. The Application of Chaos Theory in Supply Chain. Surana et al. [16] argued that supply chains should be treated as complex adaptive systems, which were characterized by nonlinear interactions and strong interdependencies between the entities. Jüttner et al. [17] commented that the chaos effect would be an important research branch of supply chain risk management because of the incomplete information. The well-known bullwhip effect is an example of such chaos. Lei et al. [18] revealed the negative impacts of the bullwhip effect in a supply chain chaos system and provided an effective method, radial-basis function neural network, to help reduce and mitigate the bullwhip effect. Ma et al. [19] investigated the complexity and bullwhip effect of a multichannel supply chain, finding that a moderate- or lowprice discount sensitivity kept the system stable, and a high price discount sensitivity brought the system into the twofold cycle or chaotic state. Besides, supply chains can display some of the key characteristics of chaotic systems. Chaos can be used as a metaphor to describe how a small change can be amplified to have a large effect on the system. In a chaos system, the variables are sensitive to initial conditions. It means that any small deviation will be magnified exponentially until there is no means of differentiating the actual signal from the signal generated by the error [20]. It finds that chaos theory principles can be used to assist in the examination of forecasting, product design, and inventory management challenges.

Chaos theory shows the potential to be a tool that can be instrumental in helping explain why unpredictability occurs within nonlinear systems. Ultimately this may assist managers in making better supply chain management decisions, benefiting organizations and customers by simultaneously enhancing cost-effectiveness and improving customer service levels [21]. Hwarng and Xie [22] investigated how variability and chaos occur in a multilevel supply chain and offered insights into how to manage relevant supply chain factors to eliminate or reduce system chaos. It found that the adjustment parameters for both inventory and supply line discrepancies should be more comparable in magnitude so that the degree of chaos in the supply chain system could be reduced. Hwarng and Yuan [23] further examined the chaos theory and its related methodology applied in the supply chain under demand uncertainty. The chaos theory has been applied in the analysis of the multichannel supply chain [24-27] and closed-loop supply chain [28, 29]. Facing the negative impacts of chaos on the supply chain, Göksu et al. [30] studied the synchronization and control of a chaotic supply chain system. Using active controllers and linear feedback controllers to synchronize and control the system, they also verified the robustness of proposed synchronization and control methods by computer simulations. Kocamaz et al. [31] controlled a chaotic system with artificial neural network (ANN) and synchronized two identical chaotic supply chains with different conditions with adaptive neuro-fuzzy inference system (ANFIS) based on controllers and showed the effectiveness of the proposed approaches.

As chaos theory is often used to make stability analysis in a dynamic nonlinear system, in this paper, the stability is measured by modeling a dynamic decision adjustment process based on chaos theory. Stability means that the 
optimal equilibrium solutions and the corresponding profits can be obtained after the decision adjustment based on a specific rule.

\section{Model Description}

We consider three decision-makers in the capacity sharing system, that is, a limited-capacity manufacturer (capacity requestor), an excess-capacity manufacturer (capacity provider), and a platform operator. The platform operator sets a service charge $c_{p}$ to maximize its profit. The capacity provider charges capacity-transfer price $p_{t}$ from the capacity requestor and pays service charge $c_{p}$ to the platform operator for $q_{t}$ per unit. There exists a co-opetitive game between the capacity requestor and the capacity provider: on the one hand, the capacity requestor cooperates with the capacity provider by ordering capacities from the latter. On the other hand, the two manufacturers make a quantity competition (Cournot competition) for the end consumers in the retail market.

We assume the capacity demand and supply are not urgent. Both the capacity provider and the capacity requestor have two choices, to seek matches actively or wait for partners. We analyze the capacity- and the demand-driven trading strategies. We also assume the capacity requestor has specific demand and the capacity provider can adjust itself to meet the specific demand. Under the capacity-driven trading strategy, the capacity requestor actively seeks a capacity provider from many capacity providers waiting for the trade, and the capacity-transfer price is determined by the order placed by the capacity requestor, that is, $p_{t}=a_{t}-q_{t}$. Under the demand-driven trading strategy, the capacity provider actively seeks a capacity requestor from many waiting capacity requestors based on its capacity, and the order depends on the prices set by the capacity provider, that is, $q_{t}=a_{t}^{\prime}-p_{t}$. To simplify the model and compare the results obtained in the two scenarios, we unify the relationship between $p_{t}$ and $q_{t}$ in two markets, that is, $a_{t}=a_{t}^{\prime}=1$. We assume the seeker can successfully make a deal for capacity sharing.

The platform operator makes a decision before others. The lack of information about others' decisions limits its decision-making process. Hence, we consider the platform operator to be bounded rational. It obtains the optimal decision by adjusting decisions in each period. Based on chaos theory, the platform operator, as well as the capacity requestor and capacity provider, may face stability problems in such a dynamic decision-making system.

As the capacity provider could share its remaining capacities after it decides on its own quantity, we consider the capacity sharing business happens after the capacity provider's quantity decision. As a result, the sequence of events is presented in Figure 2. The list of notations can be seen in Table 1.

The capacity requestor and the capacity provider's quantities are denoted by $q_{b}$ and $q_{s}$, respectively. The capacity requestor's quantity consists of two parts: one part made by itself and the other part borrowed from the capacity provider. We assume the capacity requestor to make full use of capacity $k$. As a result, $q_{b}=k+q_{t}$. We assume the consumers are indifferent between the products made by the two manufacturers. Hence, we model the market-clearing price $p$ with the following inverse demand function:

$$
p=a-b\left(q_{b}+q_{s}\right)
$$

where $a$ is the potential market size and $b$ represents the demand sensitivity coefficient. We normalize $b=1$ to simplify the analytical results.

The customer surplus utility is given as follows:

$$
C=a\left(q_{b}+q_{s}\right)-\frac{1}{2}\left(q_{b}^{2}+q_{s}^{2}+2 b q_{b} q_{s}\right)-p\left(q_{b}+q_{s}\right) .
$$

As the kinds of products vary widely, the manufacturing costs of different products are distributed over a wide range. We ignore the cost difference of the same products made by the capacity requestor and capacity provider. Hence, the capacity requestor and the capacity provider make their products with the same production cost $c$. Meanwhile, the cost of outsourcing manufacturing occurs when the capacity provider shares capacity and products for the capacity requestor. As a result, the objective functions of the capacity requestor and capacity provider are given by

$$
\begin{aligned}
& \max _{q_{t}} \pi_{b}=(p-c) k+\left(p-p_{t}\right) q_{t}, \\
& \max _{p_{t}, q_{s}} \pi_{s}=(p-c) q_{s}+\left(p_{t}-c-c_{p}\right) q_{t} .
\end{aligned}
$$

Variable costs are the costs of labor or materials that change with sales. Considering that the service cost per unit is very low, we assume the variable cost for service to be zero. The platform operator's profit function would then be

$$
\max _{c_{p}} \pi_{p}=c_{p} q_{t}
$$

\section{Analysis of Basic Models}

4.1. Capacity-Driven Case (CD Case). Under the CD case, a capacity requestor with specific demand actively seeks the capacity provider. The matching is driven by the order of the capacity requestor. The limited capacity affects the order quantities and then drives the prices. For simplicity of exposition and analytical tractability, we use a linear inverse demand function to model the transfer price as $p_{t}=1-q_{t}$. The equilibrium outcomes are given in Proposition 1. Please refer to the Appendix B for proofs of all propositions.

Proposition 1. Under $C D$ case, the equilibrium outcomes are: $c_{p}^{C D^{*}}=(1 / 4)(1-c-k), q_{t}^{C D^{*}}=(1 / 8)(1-c-k), p_{t}^{C D^{*}}=$ $(1 / 8)(7+c+k)$, and $q_{s}^{C D^{*}}=(1 / 8)(8 a-3 c-11 k-5)$.

It is reasonable that a higher production cost $c$ raises the capacity-transfer price $p_{t}$ and then lowers the amount of shared capacity $q_{t}$, which forces the platform operator to lower the service charge. The impacts of $k$ on the equilibrium outcomes are consistent with those of $c$. It is also reasonable that a higher capacity $k$ owned by the capacity requestor will 


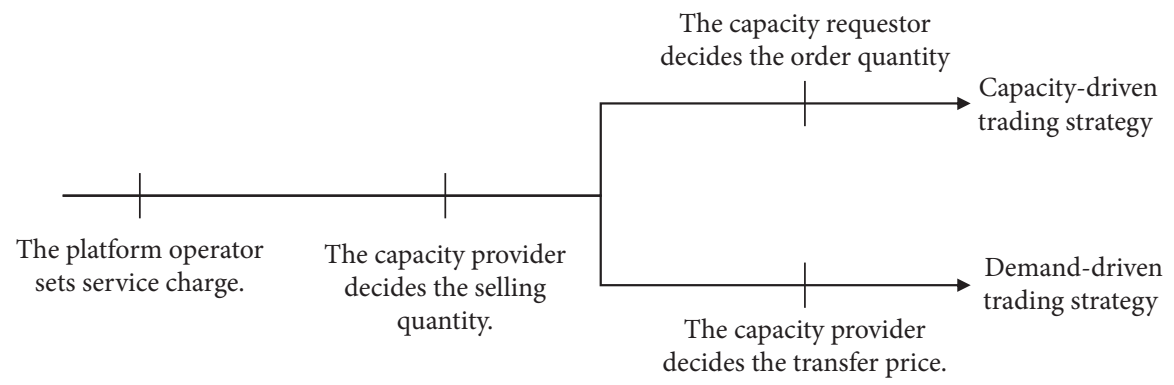

Figure 2: Sequence of events.

TABLE 1: List of notations.

\begin{tabular}{lc}
\hline Notation & Definition \\
\hline$a$ & Potential market size \\
$c$ & $\begin{array}{c}\text { Demand sensitivity coefficient } \\
g\end{array}$ \\
$k$ & $\begin{array}{c}\text { Adjustment speed } \\
p\end{array}$ \\
$q_{b}$ & $\begin{array}{c}\text { Production cost of the capacity provider and the capacity requestor } \\
q_{t}\end{array}$ \\
$p_{t}$ & Capacity quantity owned by the capacity requestor \\
$c_{p}$ & Total quantity required by the capacity requestor \\
$C$ & Capacity quantity ordered by the capacity requestor \\
$\pi_{b}$ & Unit capacity-transfer price \\
$\pi_{s}$ & Unit service charge, decision variable of the platform operator \\
$\pi_{p}$ & Customer surplus utility \\
\hline
\end{tabular}

drive it to order less capacities from others, which raises the capacity-transfer price and makes the platform operator set a lower service charge. The optimal expected profits are given in Appendix B, from which we can see the capacity requestor's profit $\pi_{b}^{\mathrm{CD}}$ increases in $k$ and the capacity provider's profit $\pi_{s}^{\mathrm{CD}}$ decreases in $k$.

Proposition 2. Under the CD case, the basic condition guaranteeing that both the capacity requestor and the capacity provider join in the capacity-sharing business is $0<c+k<1$.

Proposition 2 shows that the CD trading strategy is available for the capacity requestors with capacities to be seriously limited and costs to be relatively low. Considering the case without capacity sharing, it is not difficult to get that the capacity requestor's capacity will meet the demand when $k \geq(1 / 3)(a-c)$. Because of the cost advantages, some of the manufacturers may borrow capacities even when they have sufficient capacities. As a result, we do not treat $k>(1 / 3)(a-c)$ as a basic condition for the adoption of capacity sharing. Only when $k<1-c$ may both the capacity requestor and the capacity provider participate in the capacity sharing business with the CD trading strategy. If the capacity limit is not so serious, the capacity requestor is not willing to borrow capacity because the profits obtained from capacity sharing are not large enough to cover the cost. As for the cost $c$, only when $c<1-k$ may both traders participate in the capacity-sharing business. If $c$ is relatively large, the corresponding capacity-transfer price will be too high for the capacity requestor to bear. In a word, when the capacity limitation is relatively serious or the cost is relatively small, both the capacity requestor and the capacity provider will consider participating in the capacity-sharing business with the CD trading strategy.

Considering the bounded rationality of the platform operator, the optimal service charge can only be got after several adjustments. According to [32, 33], the platform operator uses bounded rational expectation and adopts the so-called myopic adjustment mechanism

$$
c_{p}^{\mathrm{CD}}(t+1)=c_{p}^{\mathrm{CD}}(t)+g^{\mathrm{CD}} \cdot c_{p}^{\mathrm{CD}}(t) \cdot \frac{\partial \pi_{p}^{\mathrm{CD}}(t)}{\partial c_{p}^{\mathrm{CD}}(t)},
$$

which minimizes the marginal profits of the platform operator. We can see that the adjustment stops (i.e., $\left.c_{p}^{\mathrm{CD}}(t+1)=c_{p}^{\mathrm{CD}}(t)\right)$ and the equilibrium point is obtained when the marginal profits of the platform operator are minimized to be zero. According to Jury [34], to guarantee the system CD to be stable at the equilibrium solution $c_{p}^{*}$, the condition can be expressed as follows:

$$
\left|\frac{\partial c_{p}^{\mathrm{CD}}(t+1)}{\partial c_{p}^{\mathrm{CD}}(t)}\right|<1 \Leftrightarrow-1<1+\frac{1}{4} g(-1+c+k)<1 .
$$


Given $c+k<1$, we have $(1 / 4) g(-1+c+k)<0$, and the condition can be written as $1+(1 / 4) g(-1+c+k)>-1$.

Proposition 3. Under the $C D$ case, the equilibrium point $c_{p}^{C D^{*}}$ is stable when $g(1-c-k)<8$.

Proposition 3 shows that a higher $c$, a higher $k$, and a lower $g$ are good for the system stability. Adopting CD trading strategy, those capacity requestors manufacturing high-cost goods or being with higher capacities will be more likely to keep the supply chain system stable. Intuitively, a higher adjustment speed can make the decision-maker reach the equilibrium outcome more quickly and then obtain a higher accumulated profit. It is true when the decision is far away from the optimal one. However, when the decision has been very closed to the optimal one, a higher adjustment speed may lead the decision value to fluctuate around the optimal one. Proposition 3 indicates that a relatively low adjustment speed guarantees the stability of the system. The upper limit $g_{\max }=(8 /(1-c-k))$ can measure the stability performance of the system. The larger the $g_{\max } \mathrm{CD}$ is, the better the stability performance is. Similarly, the lower limit $c_{\text {min }^{\mathrm{CD}}}=1-k-(8 / g)$ and $k_{\min ^{\mathrm{CD}}}=1-c-(8 / g)$ can be solved out.

4.2. Demand-Driven Case (DD Case). Under the DD case, a capacity provider tries to actively seek and match a capacity requestor based on its specific supply capacity. The capacity provider holds the pricing power, and the demand is driven by the price. For analytical tractability and comparability with the $\mathrm{CD}$ case, we use a linear demand function to model the order as $q_{t}=1-p_{t}$. By backward induction, in stage 3, due to $\left(\partial^{2} \pi_{s} / \partial p_{t}^{2}\right)=-2$, there exists a unique optimal $p_{t}$. The best response function (BRF) of $p_{t}$ is

$$
p_{t}=\frac{1}{2}\left(1+c+c_{p}+q_{s}\right)
$$

Then the BRF of $q_{t}$ is

$$
q_{t}=\frac{1}{2}\left(1-c-c_{p}-q_{s}\right)
$$

In stage 2 , the capacity provider decides on its own quantity. Due to $\left(\partial^{2} \pi_{s} / \partial q_{s}^{2}\right)=-(3 / 2)$, the BRF of $q_{s}$ is

$$
q_{s}=\frac{1}{3}\left(-1+2 a-c+c_{p}-2 k\right) \text {. }
$$

In stage 1 , the platform operator determines the service charge. A perfect rational decision-maker can get the unique optimal $c_{p}^{*}$ since $\left(\partial^{2} \pi_{p} / \partial c_{p}^{2}\right)=-(4 / 3)$. Substituting $c_{p}^{*}$ into the BRFs of $q_{t}, p_{t}, q_{s}$, we have the equilibrium outcomes under DD case, as shown in Proposition 4.

Proposition 4. Under $D D$ case, the equilibrium outcomes are: $c_{p}^{D D^{*}}=(1 / 4)(2-a-c+k), q_{t}^{D D^{*}}=(1 / 6)(2-a-c+k)$, $p_{t}^{D D^{*}}=(1 / 6)(4+a+c-k), \quad$ and $\quad q_{s}^{D D^{*}}=(1 / 12)$ $(7 a-5 c-7 k-2)$.
The impact of the production cost $c$ on the equilibrium outcomes under DD case is similar to those under CD. The difference is that the influence of $k$ on the equilibrium outcomes under the two models. The reason is that in this market, the amount of shared capacity is driven by the price. The capacity $k$ affects the price and then indirectly affects the quantity. The capacity requestor with higher capacity has a larger power bargaining with the capacity provider and then gets a lower capacity-transfer price. For the capacity provider, it can set a higher price for the capacity requestors with capacity limitations to be more serious. The capacitytransfer price further affects the quantities and the service charge.

Proposition 5. Under the DD case, the basic condition guaranteeing that both the capacity requestor and the capacity provider join in the capacity-sharing business is $a+c<2+k$.

Proposition 5 indicates that DD trading strategy is available for the low-cost capacity requestors with sufficiently large capacities, that is, $c<2-a+k$ or $k>a+c-2$. If $k<a+c-2$, the capacity requestor will not consider DD as an option. It makes sense that the capacity requestor facing serious capacity limitation cannot wait for the capacity provider. If $k<\min \{a+c-2,1-c\}$, it will actively seek matches.

Similarly, the bounded rational platform operator adjusts service charge under the DD case following the below rule:

$$
c_{p}^{\mathrm{DD}}(t+1)=c_{p}^{\mathrm{DD}}(t)+g^{\mathrm{DD}} \cdot c_{p}^{\mathrm{DD}}(t) \cdot \frac{\partial \pi_{p}^{\mathrm{DD}}(t)}{\partial c_{p}^{\mathrm{DD}}(t)} .
$$

The condition guaranteeing the system to be stable at the equilibrium solution $c_{p}^{*}$ is

$$
\left|\frac{\partial c_{p}^{\mathrm{DD}}(t+1)}{\partial c_{p}^{\mathrm{DD}}(t)}\right|<1 \Leftrightarrow-1<1+\frac{1}{3} g(-2+a+c-k)<1 .
$$

Given $a+c<2+k$, the condition (11) can be written as $1+(1 / 3) g(-2+a+c-k)>-1$.

Proposition 6. Under the DD case, the equilibrium point $c_{p}^{D D^{*}}$ is stable when $g(2+k-a-c)<6$.

Proposition 6 indicates that under the DD case, a lower $g$ and $k$ and a higher $a$ and $c$ are good for the system stability. Different from the $\mathrm{CD}$ case, the platform operator may prefer the capacity requestor with lower capacities, when it can use a higher decision adjustment speed $g$ without losing the system stability. In order to keep the system stable, the platform operator may make the low-capacity requestors adopt DD trading strategy, make those high-capacity requestors adopt $\mathrm{CD}$ trading strategy. The upper limit $g_{\max }=(6 /(2+k-a-c)), k_{\max }=a+c+(6 / g)-2$, and the lower limit $c_{\min ^{\mathrm{DD}}}=2+k-a-(6 / g)$ can be solved out. 


\section{Trade-off between Profitability and Stability}

In this section, we compare the performance of systems CD and DD on stability and profitability in order to study the choice of trading strategy. According to Propositions 2 and 5 , the one who can make a choice between $\mathrm{CD}$ and $\mathrm{DD}$ should meet the basic condition given as follows:

$$
\begin{aligned}
& c+k<1, \\
& a+c<2+k .
\end{aligned}
$$

Note that $c_{\max }=\min \{1-k, 2+k-a\}$, only the firms with production cost satisfying $c<c_{\max }$ and the limited capacity requestor with $a+c-2<k<1-c$ have two choices of CD and DD. In this section, we pay more attention to the decision of the platform operator on CD and DD based on the assumption that both the capacity provider and capacity requestor meet the condition (12) and accept the arrangement of the platform operator. The assumption is relaxed in Section 6 for the study on how all the decision-makers freely choose trading strategies.

5.1. Comparison of Profitability. In this paper, profitability is measured by the amount of profits obtained under different trading strategies. That is the maximum profit theoretically obtained by a decision-maker. If all the decision-makers are perfectly rational, the equilibrium solutions in Propositions 1 and 4 can be obtained. Comparing the expected profits obtained under DD and CD trading strategies, we can get the platform operator's preference on trading strategies presented in Proposition 7.

Proposition 7. Under the assumption of perfect rationality, there exist thresholds $c_{t h}^{\pi_{p}}$ such that the following occurs:

$$
\begin{cases}\pi_{p}^{\mathrm{CD}}>\pi_{p}^{\mathrm{DD}}, & \text { if } \max \left\{0, c_{\mathrm{th}}^{\pi_{p}}\right\}<c<c_{\max }, \\ \pi_{p}^{\mathrm{CD}}<\pi_{p}^{\mathrm{DD}}, & \text { if } 0<c<\min \left\{c_{\mathrm{th}}^{\pi_{p}}, c_{\max }\right\} .\end{cases}
$$

Proposition 7 indicates that a profit-maximizing platform operator would like to make the high- and low-cost firms adopt $\mathrm{CD}$ and $\mathrm{DD}$ trading strategy, respectively. If $-1+a-2 k<0$, we have the threshold $c_{\mathrm{th}}^{\pi_{p}}>c_{\max }$, and then $\pi_{p}^{\mathrm{CD}}<\pi_{p}^{\mathrm{DD}}$ is always true with $c \in\left(0, c_{\max }\right]$. Similarly, if $2 a+\sqrt{3}>(\sqrt{3}+2) k+4$, we have $c_{\text {th }}^{\pi_{p}}<0$, and $\pi_{p}^{\mathrm{CD}}>\pi_{p}^{\mathrm{DD}}$ is always true. For example, making $a=1.5$ and $k=0.2$, we have $c_{\text {th }}^{\pi_{p}}=0.05$ and $c_{\max }=0.7$, and the platform operator prefers to make the firms with $c<0.05$ accept DD and those with $0.05<c<0.7$ accept $C D$, as shown in Figure 3(a). Making $a=2$ and $k=0.2$, we have $c_{\text {th }}^{\pi_{p}} \approx-3.68<0$, and the profits obtained by $\mathrm{CD}$ is always larger than that by $\mathrm{DD}$, as shown in Figure 3(b). Making $a=1.2$ and $k=0.2$, we have $-1+a-2 k<0, c_{\text {th }}^{\pi_{p}} \approx 2.29$, and $c_{\max }=0.8, c_{\text {th }}^{\pi_{p}}>c_{\max }$, and then $\pi_{p}^{\mathrm{CD}}<\pi_{p}^{\mathrm{DD}}$ is always true, as shown in Figure $3(\mathrm{c})$.

We only consider the most interesting case $0<c_{\text {th }}^{\pi_{p}}<c_{\text {max }}$. In general, the platform operator is very important for the trading and even monopoly the capacity-sharing markets, making the capacity requestor and capacity provider accept the trading strategies set by the platform operator. For example, as a platform operator, the INDICS platform (http://www.indics.com/) provides a capacity sharing platform for the capacity requestor and capacity provider to match each other. And the platform can induce their choices by some specific methods. In the following paper, we assume that the platform operator leads the choice of trading strategies and both the capacity requestor and the capacity provider accept the decisions. As a result, we are more concerned about the decisions of the platform operator. In the next section, we consider a more general case in which the platform operator is bounded rational. The choice of a trading strategy will be more complex for the platform operator, as well as the capacity requestors and capacity providers, considering the stability of the system.

5.2. Comparison of Stability. We compare the stabilities of two systems (denoted by CD and DD) according to Propositions 3 and 6 . More specifically, we measure the stabilities by the thresholds (i.e., $g_{\max } \mathrm{DD}, g_{\max }{ }^{\mathrm{CD}}, c_{\min ^{\mathrm{DD}}}$, and $c_{\min ^{\mathrm{CD}}}$ ), which divide the system into stable and unstable states. For example, from the perspective of $g$, the system DD is stable if $0<g<g_{\max ^{\mathrm{DD}}}$. The system CD is stable if $0<g<g_{\max }{ }^{\mathrm{CD}}$. The system CD has better stability than DD if $g_{\max }>g_{\max }$ because the System CD has a larger range of $g$ keeping the system stable. Similarly, from the perspective of $c$, the system under CD trading strategy is stable if $c_{\min ^{C D}}<c<1-k$. The system under DD trading strategy is stable if $c_{\min ^{\mathrm{DD}}}<c<2+k-a$. The system $\mathrm{CD}$ has a better stability than DD if $c_{\min }{ }^{\mathrm{CD}}<c_{\min } \mathrm{DD}$. As the relationship between $g_{\max }$ and $g_{\max }$ is also influenced by the parameters $c, a$, and $k$, we can compare the stability of two systems by comparing $g_{\max }{ }^{\mathrm{CD}}$ with $g_{\max }{ }^{\mathrm{DD}}$ from the perspective of $c$, which is given in Proposition 8.

Proposition 8. Under the assumption of bounded rationality, there exists a threshold $c_{\text {th }}^{g_{\max }}$ such that the following occurs:

$$
\begin{cases}g_{\max }{ }^{\mathrm{CD}}>g_{\max } \mathrm{DD} & \text { if } 0<c<\min \left\{c_{\mathrm{th}}^{g_{\max }}, c_{\max }\right\}, \\ g_{\max }{ }_{\operatorname{mD}}<g_{\max }, & \text { if } \max \left\{0, c_{\mathrm{th}}^{g_{\max }}\right\}<c<c_{\max } .\end{cases}
$$

For the high-cost firms, accepting DD leaves better stability for the platform operator than accepting CD. The bounded rational platform operator is suggested to recommend the firms with high production costs to adopt DD trading strategy for better stability performance and recommend the firms with low production costs to adopt CD trading strategy.

If the capacity requestor's capacity is sufficiently larger, that is, $k>((a-1) / 2)$, we have $\left\{c_{\mathrm{th}}^{g_{\max }}>c_{\max }\right\}$, and the system $\mathrm{CD}$ always has better stability than DD. As the capacity is limited when $k<((a-1) / 3)$, this case only happens on the firm with sufficient capacity borrowing capacity from others. For the firms facing a serious capacity problem, that is, $k<(1 / 7)(4 a-5)$, we have $c_{\text {th }}^{g_{\max }}<0$. For these firms, DD 


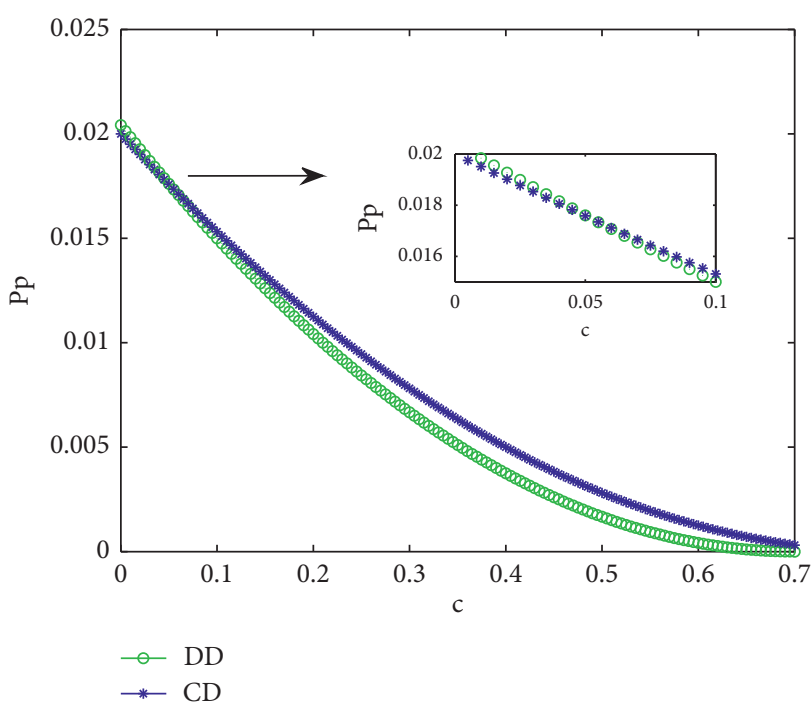

(a)

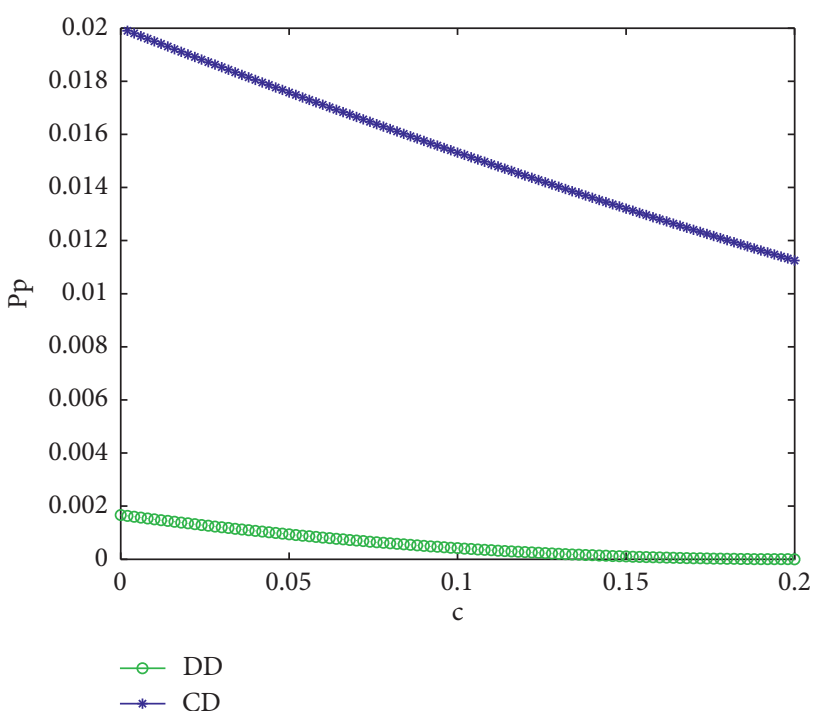

(b)

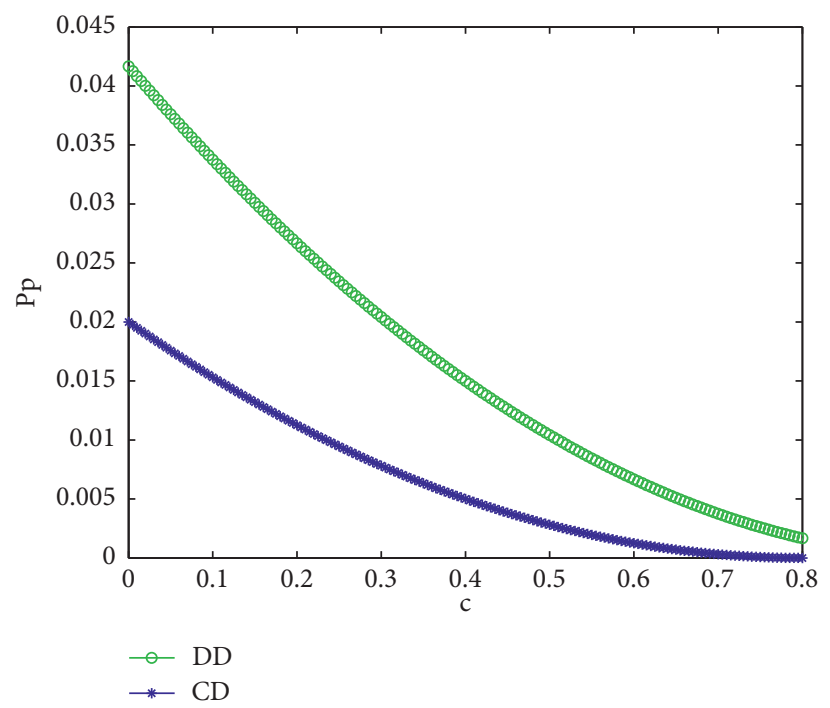

(c)

Figure 3: Profit comparison: (a) $a=1.5$ and $k=0.2$; (b) $a=2.0$ and $k=0.2$; and (c) $a=1.2$ and $k=0.2$.

always has better stability than $\mathrm{CD}$, regardless of how much the production cost is.

Making numerical simulations helps better understand the importance of stability and the difference between the two systems. Setting default values of the parameters $a, c, k$, and $g$ as shown in Table 2, we have $c_{\max }=0.7$, and only the firms with $c<0.7$ can participate in capacity sharing under DD and CD trading strategies according to the basic condition equation (12). We can also get the thresholds determining whether or not the system CD or DD is stable, which are also shown in Table 2.

Actually, in an unstable system, the decision-maker could neither reach the equilibrium nor obtain maximum profit. Take the CD case for example. As shown in Table 2, we can see that the threshold $g_{\max }=13.33$ and any $g<g_{\max } \mathrm{CD}$ makes the system stable. As a result, we make $g=$ $11,14,17$ to build the stable, period-doubling, and chaos system and then draw the time series of $c_{p}, q_{s}$, and $q_{t}$ with respect to $g$ to show the decision adjustment processes in different systems, as shown in Figure 4.

Observe that, when $g=11$, the system is stable, and the decision can be stably optimized after several adjustments. When $g=14$, the system is double-cycle, and the decision sways between the peaks and troughs. When $g=17$, the system is chaos, and the decision seems to be disordered after several adjustments.

To better show how the parameters $g, c$, and $k$ affect the optimal decisions and the difference in stability performance of systems CD and DD, we draw the bifurcation diagrams shown in Figure 5, which shows the equilibrium decision $c_{p}$ after several adjustments and iterations with respect to $g, c$, and $k$.

Take Figure 5(a) for example. First, when $g$ stays at a relatively low level (i.e., lower than $g_{\max }$ DD or $g_{\max }$ ),$c_{p}$ 
TABLE 2: Values of the capacity-sharing system parameters.

\begin{tabular}{lcccccccccccc}
\hline Parameters & $a$ & $c$ & $k$ & $g$ & $c_{\min ^{\mathrm{DD}}}$ & $c_{\min ^{\mathrm{CD}}}$ & $g_{\max ^{\mathrm{DD}}}$ & $g_{\max ^{\mathrm{CD}}}$ & $c_{\min ^{\mathrm{DD}}}$ & $c_{\min ^{\mathrm{CD}}}$ & $k_{\max } \mathrm{DD}$ & $k_{\min ^{\mathrm{CD}}}$ \\
\hline Values & 1.5 & 0.2 & 0.2 & 20.0 & 0.4 & 0.4 & 12.0 & 13.3 & 0.2 & 0.1 & 0.0 & 0.4 \\
\hline
\end{tabular}

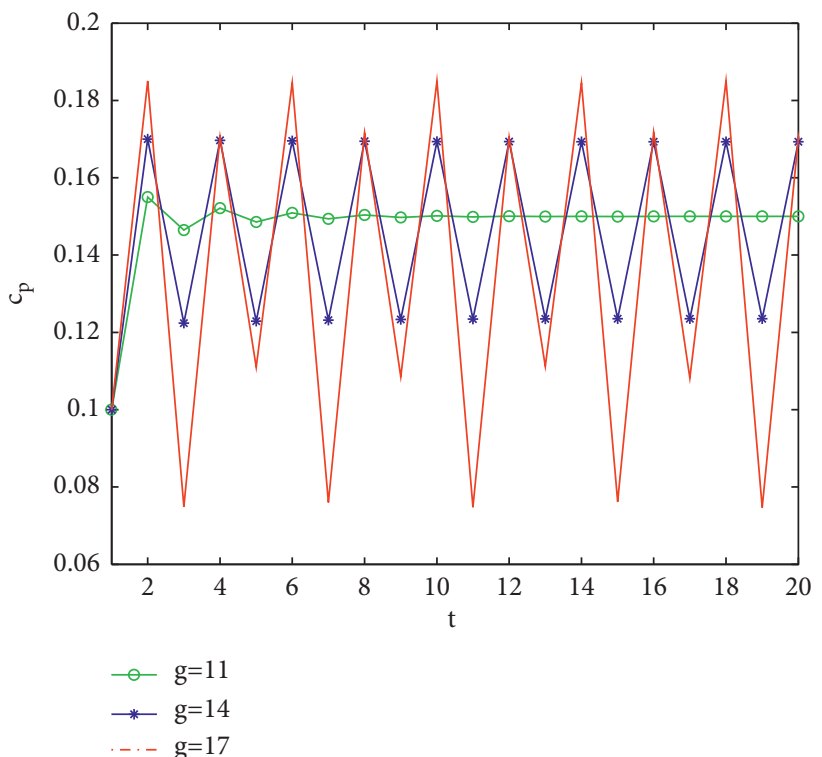

(a)

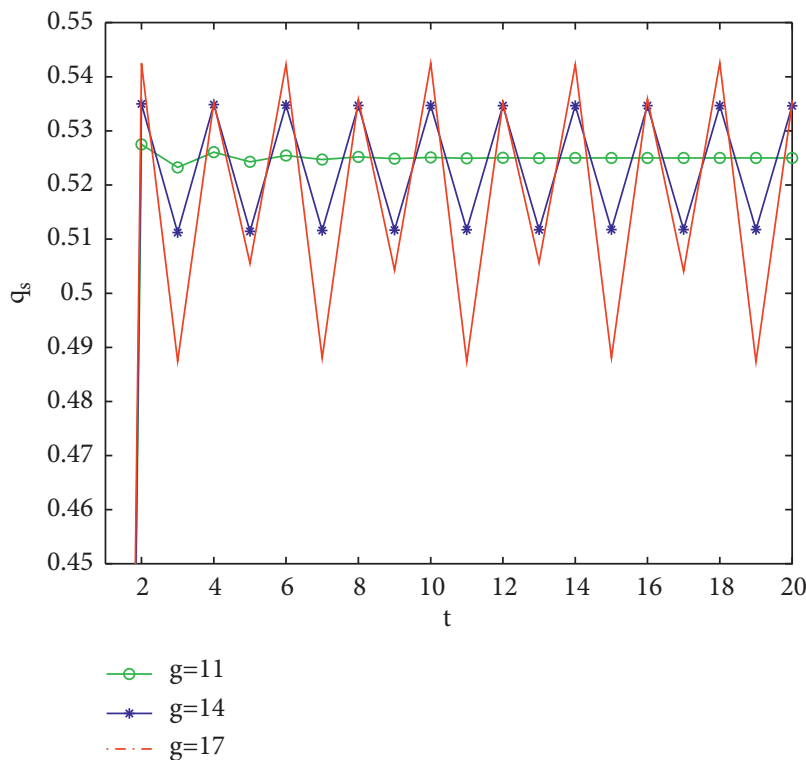

(b)

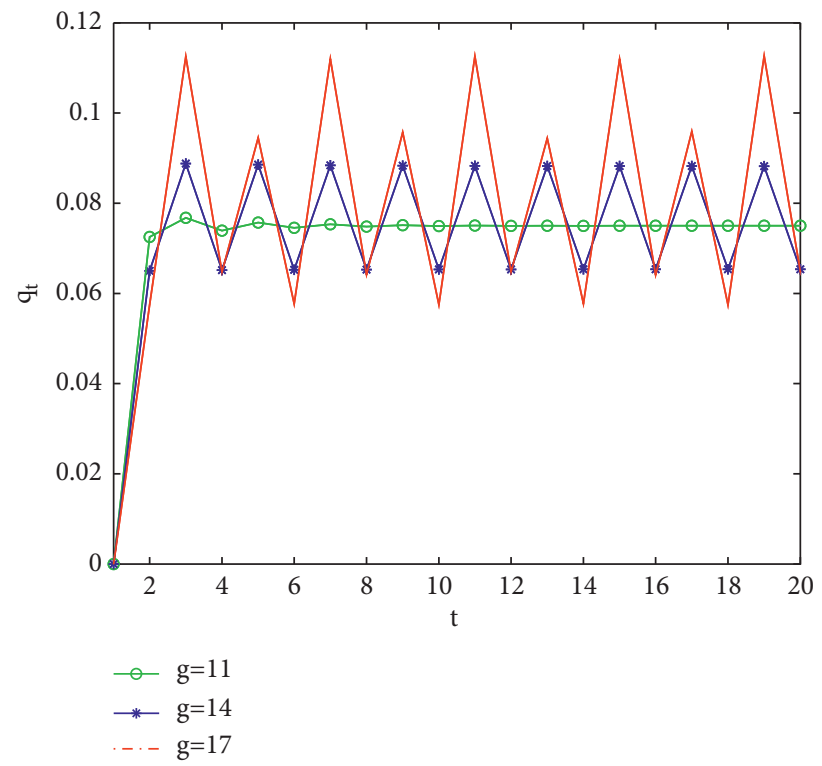

(c)

Figure 4: Time series for different states $(g=11,14,17)$ under CD: (a) $c_{p}$, (b) $q_{s}$, and (c) $q_{t}$.

tends to be a unique optimal solution. When $g$ is larger than the thresholds $g_{\max }$ DD $\left(\right.$ or $g_{\max }$ ), the decision-maker cannot obtain any unique solution. Instead, the decisions will swing between two or more values at both sides of the optimal one. If $g$ increases to be larger than a threshold, the system will go into chaos, and the decision $c_{p}$ will become chaotic. We can also see the different performance of stability between systems CD and DD. As $g_{\max }{ }^{\mathrm{DD}}<g_{\max }{ }^{\mathrm{CD}}$ shown in Table 2, the system $\mathrm{CD}$ can be stable with a larger range $g \in\left(g_{\max ^{\mathrm{DD}}}, g_{\max }\right)$ comparing with the system DD.

Apart from the platform operator, the capacity requestor and the capacity provider cannot get stable decisions either in an unstable system. Driven by the service charges, the decisions of the capacity requestor and capacity provider may also change from being stable to unstable. Figure 6 shows that the decisions of the capacity requestor and 


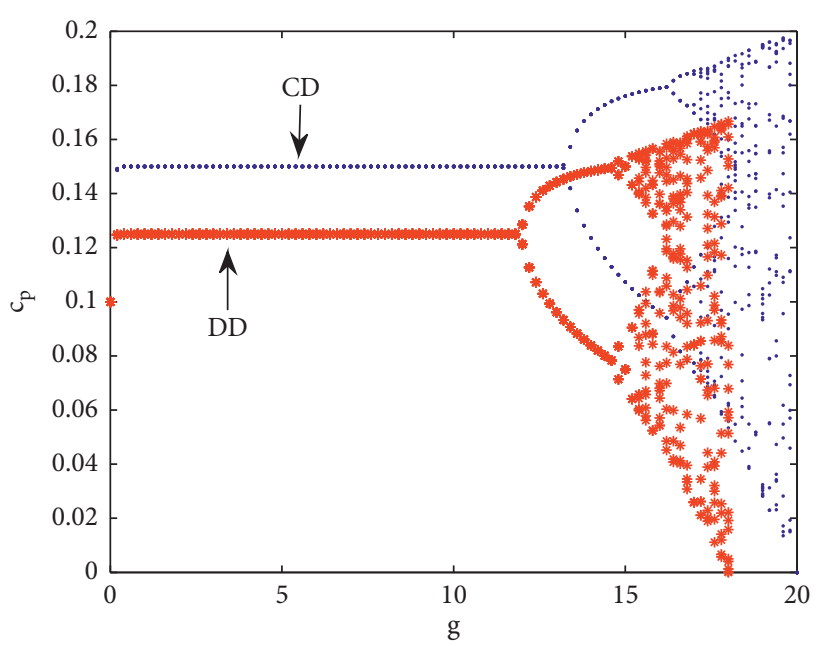

(a)

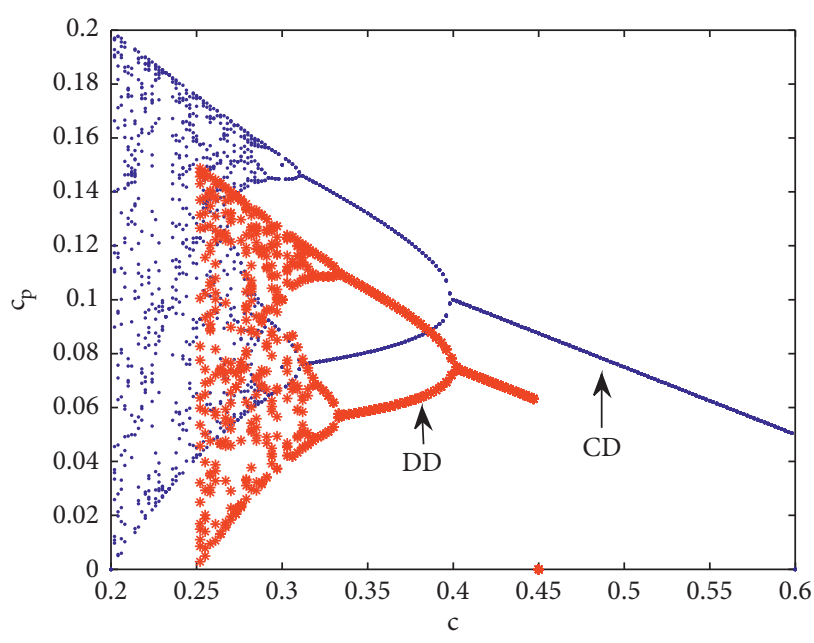

(b)

FIgURE 5: Bifurcation diagrams for the decisions of the platform operator: (a) $c_{p}$ w.r.t. $g$ and (b) $c_{p}$ w.r.t. $c$.

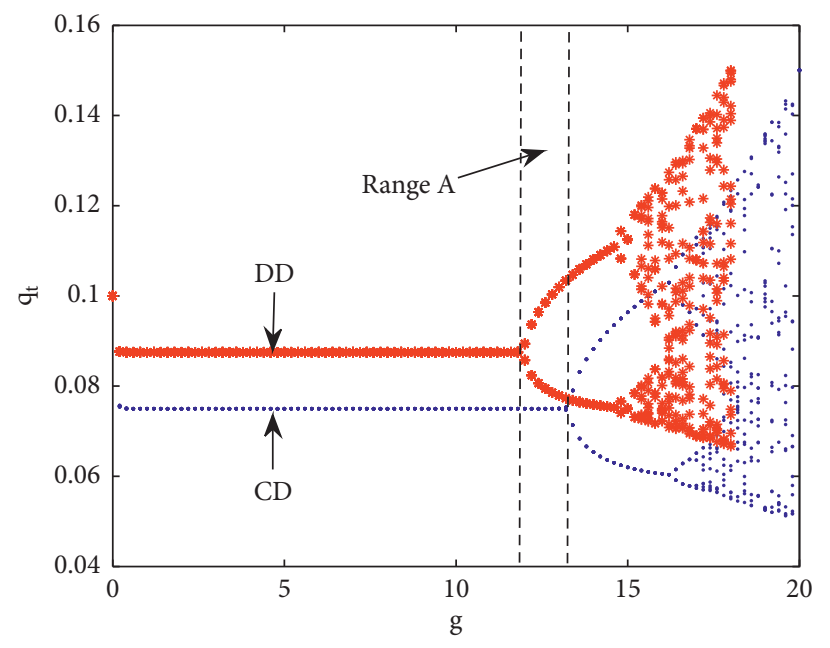

(a)

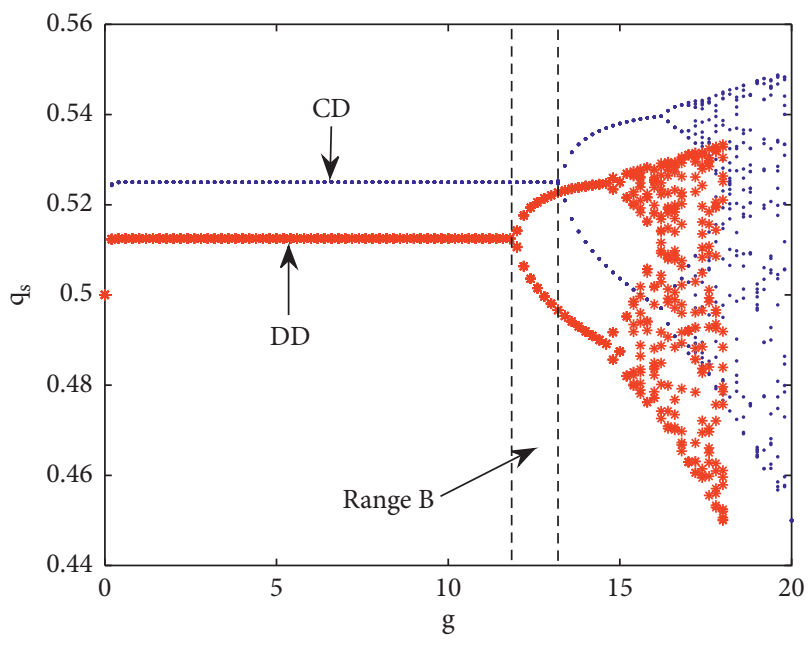

(b)

FiguRe 6: Bifurcation diagrams for the decisions of the capacity requestor and the capacity provider: (a) $q_{t}$ w.r.t. $g$ and (b) $q_{s}$ w.r.t. $g$.

capacity provider will also be influenced by the parameters $g$ and $c$. The bifurcation points of $q_{t}$ and $q_{s}$ are consistent with those of $c_{p}$.

From Figures 4-6, we can see that only in a stable system can the platform operator and the capacity requestor and the capacity provider get unique optimal solutions. Observing the ranges A and B in Figure 6, although DD may be a profitmaximizing trading strategy, it may not be chosen if $g$ locates in these two ranges. This leads to the study on the trade-off between profitability and stability.

5.3. Trade-Off. Interestingly, Propositions 7 and 8 indicate that the platform operator can get higher expected profits making the high-cost capacity requestor and capacity provider accept $C D$ trading strategy, while this may cause a worse stability performance of system $\mathrm{CD}$. The profitability and stability of the system are contradictory from the perspective of $c$. Stability is much more important than profitability as we have proved that the platform operator can neither reach equilibrium nor obtain optimal expected profits in an unstable system. In short, the decision-making principle is to follow the order of the objects as follows:

The maximum profit in a stable system $>$ the submaximum profit in a stable system $>$ the maximum profit in an unstable system $>$ the submaximum profit in an unstable system.

It is not difficult to understand that "the maximum profit in a stable system" is always better than "the submaximum profit in an unstable system." Why should the platform choose "the submaximum profit in a stable system" rather than "the maximum profit in an unstable system"? To answer this question, we have introduced the negative impact of unstable factors on decisions and profits. Besides, we also 
need to give analytical decision support for the platform. According to Propositions 7 and 8, the trade-off between profitability and stability is closely related to production cost. Hence, the order of the thresholds of $c$ is very important for the choice of trading strategies. According to the order of these thresholds, we present four scenarios shown in Table 3.

The final decisions may be quite different due to the different orders of the thresholds in each scenario. As the order of these thresholds depends on $k$ and $a$, we present four scenarios with respect to $k$ and $a$, as shown in Table 4 .

The order of thresholds of $c$ depends on the level of capacity $k$. Observing that, when the capacity is seriously limited, that is, $k_{1}<k<k_{3}$, we have scenario 1 in which the system DD has a better stability performance than CD, that is, $c_{\min ^{\mathrm{DD}}}<c_{\min ^{\mathrm{CD}}}$. If $k$ increases to be located in $\max \left\{k_{1}, k_{3}\right\}<k<k_{2}$, system CD has a better stability performance than DD, as shown in scenario 2 . As a result, the threshold $\max \left\{k_{1}, k_{3}\right\}$ determines whether which system has a better stability performance. In these two scenarios, the thresholds $c_{\min ^{\mathrm{DD}}}$ and $c_{\min ^{\mathrm{CD}}}$ locate in the area where the optimal expected profits $\pi_{p}^{\mathrm{CD}}>\pi_{p}^{\mathrm{DD}}$. If $k$ increases to be $k_{1}<k<\min \left\{k_{4}, k_{5}\right\}$, as shown in scenario 3 , the thresholds $c_{\min }{ }^{\mathrm{DD}}$ and $c_{\min }{ }^{\mathrm{CD}}$ will locate on each side of the threshold $c_{\mathrm{th}}^{\pi_{p}}$. In scenario $4, k$ increases to be $k_{4}<k<k_{5}$, and both the thresholds $c_{\min ^{\mathrm{DD}}}$ and $c_{\min }{ }^{\mathrm{CD}}$ locate in the area where the optimal expected profits $\pi_{p}^{\mathrm{DD}}>\pi_{p}^{\mathrm{CD}}$. Considering the profitability and stability of the $\mathrm{CD}$ and $\mathrm{DD}$, we have the optimal solutions in different scenarios based on the decisionmaking principle, as shown in Table 5.

Proposition 9. The best choices of trading strategy for the platform are presented in Table 5.

In Table 5, there is neither a satisfactory solution nor an optimal solution for the "unstable" cases in which the production cost is relatively low. For the case with "maximum, CD (or DD)," the final decision is CD (or DD), and the decision maximizes the profit, meaning that the decision $\mathrm{CD}$ (or DD) has better profitability and stability compared with DD (or CD). For the case with "submaximum, CD (or $\mathrm{DD})$," the final decision is CD (or DD), which is not an optimal solution but a satisfactory one guaranteeing the system stability and higher accumulated profits.

Take the submaximum case in scenario 3 in Table 5 for example. We need to build the condition $c_{\min }{ }^{\mathrm{CD}}<c<c_{\text {th }}^{\pi_{p}}$ in scenario 3. Making $a=1.5, g=16$, we have $a_{3} \approx 2.05$ and $a<a_{3}$. Observe in Table 4. We need to build $k_{2}<k<k_{4}$. We then obtain $k_{2}=0.217$ and $k_{4}=0.221$ and set $k=0.218$ to satisfy $k_{2}<k<k_{4}$ and match scenario 3 according to Table 4 . Then we have $c_{\mathrm{th}}^{\pi_{p}^{4}} \approx 0.304, c_{\min ^{\mathrm{DD}}}=0.343$, and $c_{\mathrm{min}_{\mathrm{r}}^{\mathrm{CD}}}=0.282$. Set $c=0.29$ to meet the condition $c_{\min ^{\mathrm{CD}}}<c<c_{\text {th }}$. Based on Proposition 8, we know that when $c<c_{\text {th }}^{\pi_{p}}$, the optimal expected profits $\pi_{p}^{\mathrm{CD}}<\pi_{p}^{\mathrm{DD}}$. However, as shown in Table 5 , the final decision is $\mathrm{CD}$ because of the system stability. Only in the stable system can the platform get stable profits. Figure 7 shows why CD is chosen by the decision-maker, in which we substitute the values above into the model and draw accumulated profits obtained in stable system CD and unstable system DD. A closer look at line DD reveals the fluctuation
TABLE 3: Four scenarios and the corresponding order of the thresholds of $c$.

\begin{tabular}{lc}
\hline Scenario & The order of the thresholds of $c$ \\
\hline 1 & $0<c_{\text {th }}^{\pi_{p}}<c_{\min ^{\mathrm{DD}}}<c_{\min ^{\mathrm{CD}}}<c_{\max }$ \\
2 & $0<c_{\mathrm{th}}^{\pi_{p}}<c_{\min ^{\mathrm{CD}}}<c_{\min ^{\mathrm{DD}}}<c_{\max }$ \\
3 & $0<c_{\min ^{\mathrm{CD}}}<c_{\text {th }}<c_{\min _{\pi_{p}}}^{\mathrm{DD}}<c_{\max }$ \\
4 & $0<c_{\min ^{\mathrm{CD}}}^{\mathrm{CD}}<c_{\min ^{\mathrm{DD}}}<c_{\mathrm{th}}<c_{\max }$ \\
\hline
\end{tabular}

caused by the decisions fluctuating at both sides of the optimal one in each decision-making cycle.

The above discussion has revealed that the thresholds $c_{\min }{ }^{\mathrm{CD}}$ and $c_{\min }{ }^{\mathrm{DD}}$ are very important for the choice of trading strategies considering the stability of the system. A lower $\min \left\{c_{\min }{ }^{\mathrm{CD}}, c_{\min }{ }^{\mathrm{DD}}\right\}$ is beneficial for the system's stability. Observe that $\left(\partial c_{\min ^{\mathrm{CD}}} / \partial g\right)=\left(8 / g^{2}\right)>0$ and $\left(\partial c_{\min ^{\mathrm{DD}}} / \partial g\right)=\left(6 / g^{2}\right)>0$. The platform operator can lower the threshold $\min \left\{c_{\min ^{\mathrm{CD}}}, c_{\min ^{\mathrm{DD}}}\right\}$ by slowing down $g$. When $g=(8 /(1-k))$, we have $c_{\min }{ }^{\mathrm{CD}}=0$, and then the system will be always stable under $\mathrm{CD}$ trading strategy; when $g=(6 /(2-a+k))$, we have $c_{\min ^{\mathrm{DD}}}=0$, and then the system will be always stable under the DD trading strategy. If the platform operator sets $g=\min \{(8 /(1-k)),(6 /(2-a+k))\}$, it can make a decision just by taking the profitability into consideration. Although a lower $g$ is beneficial for the system stability, it also affects the accumulated profits of the decision-maker because a lower $g$ will delay the time reaching the optimal decision. In Figure 8, we present the impacts of $g$ on accumulated profits.

According to Figure 8(a), comparing the lines with $g=$ $g_{\max }=13.3$ and $g=19$, if a lower $g$ is necessary for the system stability, it keeps the system stable and results in higher accumulated profits. Comparing the lines with $g=$ $g_{\max }=13.3$ and $g=0.1$, if both $g$ keep the system stable, a higher one helps reach the optimal solution more quickly and results in higher accumulated profits. Similar conclusions can be made for the DD case, as shown in Figure 8(b).

\section{Extension: The All-Win Situation}

In this section, we relax the assumption of absolute acceptance of the capacity requestor and the capacity provider and consider that they can freely choose from CD and DD or leaving from capacity sharing. To simplify the model, we consider a special case in which the capacity requestor does not have any capacity, that is, $k=0$. It is very common to see these firms in reality. For example, Nike does not own any manufacturing facilities and outsource its production. Contract factories in Vietnam, China, and Indonesia manufacture approximately $43 \%, 28 \%$, and $25 \%$ of total Nike brand footwear, respectively (https://csimarket.com/stocks/ suppliers_glance.php?code=NKE). Besides, we assume the platform adjusts the service charge with a sufficiently low speed. That is, the system can be stable so that the platform can make decisions just for better profitability. In this special case, the basic condition for both the capacity requestor and the capacity provider participating in $\mathrm{CD}$ and $\mathrm{DD}$ is $0<c<1$ and $0<a+c<2$. 
TABLE 4: Four scenarios and the corresponding parameter value range.

\begin{tabular}{|c|c|c|c|c|c|c|}
\hline & $k_{1}<k<k_{3}$ & $k_{1}<k<k_{2}$ & $k_{3}<k<k_{2}$ & $k_{2}<k<k_{5}$ & $k_{2}<k<k_{4}$ & $k_{4}<k<k_{5}$ \\
\hline$a \leq a_{1}$ & Scenario 1 & & Scenario 2 & & & \\
\hline$a_{1}<a<a_{2}$ & & Scenario 2 & & & & \\
\hline$a<a_{3}$ & & & & & Scenario 3 & Scenario 4 \\
\hline$a_{3}<a<a_{2}$ & & & & Scenario 3 & & \\
\hline
\end{tabular}

Note. The exact expressions of $k_{1}, k_{2}, k_{3}, k_{4}$, and $k_{5}$ are given in Appendix A.

TABLE 5: The final decisions and the corresponding parameter value range in four scenarios.

\begin{tabular}{|c|c|c|c|c|}
\hline Scenario 1 & $\begin{array}{c}0<c<c_{\text {th }}^{\pi_{p}} \\
\text { Unstable }\end{array}$ & $\begin{array}{c}c_{\mathrm{th}}^{\pi_{p}}<c<c_{\min ^{\mathrm{DD}}} \\
\text { Unstable }\end{array}$ & $\begin{array}{c}c_{\min ^{\mathrm{DD}}}<c<c_{\min }^{\mathrm{CD}} \\
\text { Submaximum, DD }\end{array}$ & $\begin{array}{l}c_{\min } \mathrm{CD}<c<c_{\max } \\
\text { Maximum, CD }\end{array}$ \\
\hline Scenario 2 & $\begin{array}{c}0<c<c_{\text {th }}^{\pi_{p}} \\
\text { Unstable }\end{array}$ & $\begin{array}{c}c_{\mathrm{th}}^{\pi_{p}}<c<c_{\min ^{\mathrm{CD}}} \\
\text { Unstable }\end{array}$ & $\begin{array}{c}c_{\min ^{\mathrm{CD}}}<c<c_{\min ^{\mathrm{DD}}} \\
\text { Maximum, CD }\end{array}$ & $\begin{array}{l}c_{\min } \mathrm{DD}<c<c_{\max } \\
\text { Maximum, CD }\end{array}$ \\
\hline Scenario 3 & $\begin{array}{c}0<c<c_{\min }{ }^{\mathrm{CD}} \\
\text { Unstable }\end{array}$ & $\begin{array}{c}c_{\min }{ }^{\mathrm{CD}}<c<c_{\text {th }}^{\pi_{p}} \\
\text { Submaximum, CD }\end{array}$ & $\begin{array}{c}c_{\mathrm{th}}^{\pi_{p}}<c<c_{\min }^{\mathrm{DD}} \\
\text { Maximum, CD }\end{array}$ & $\begin{array}{l}c_{\min } \mathrm{DD}<c<c_{\max } \\
\text { Maximum, CD }\end{array}$ \\
\hline Scenario 4 & $\begin{array}{c}0<c<c_{\min }{ }^{\mathrm{CD}} \\
\text { Unstable }\end{array}$ & $\begin{array}{c}c_{\min } \mathrm{CD}<c<c_{\min } \mathrm{DD} \\
\text { Submaximum, CD }\end{array}$ & $\begin{array}{c}c_{\min }^{\mathrm{DD}}<c<c_{\mathrm{th}}^{\pi_{p}} \\
\text { Maximum, DD }\end{array}$ & $\begin{array}{c}c_{\text {th }}^{\pi_{p}}<c<c_{\max } \\
\text { Maximum, CD }\end{array}$ \\
\hline
\end{tabular}

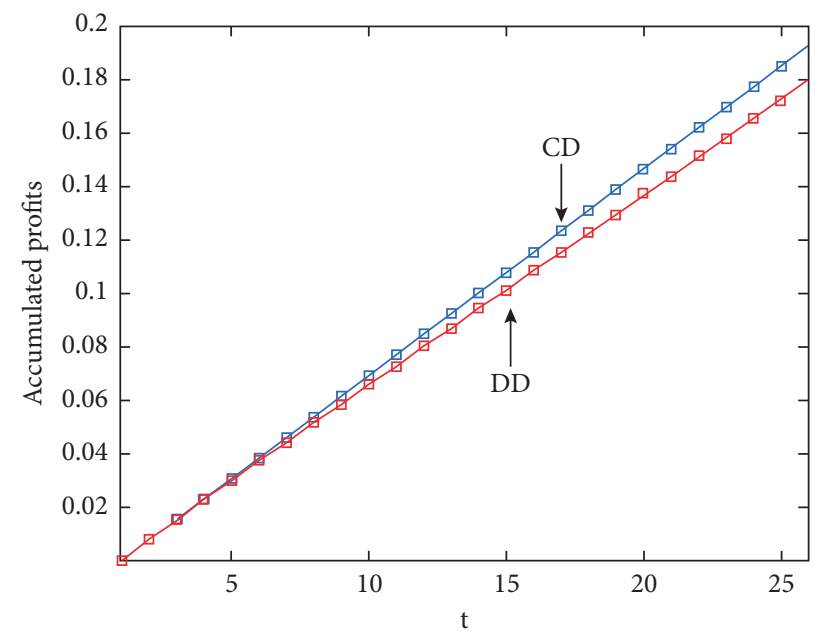

FIGURE 7: Accumulated profits of the platform operator obtained in systems CD and DD.

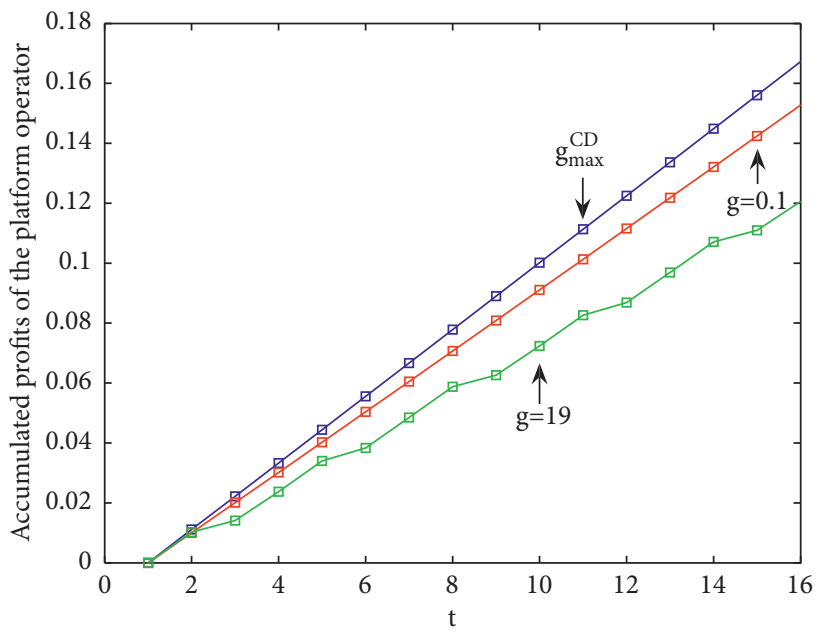

(a)

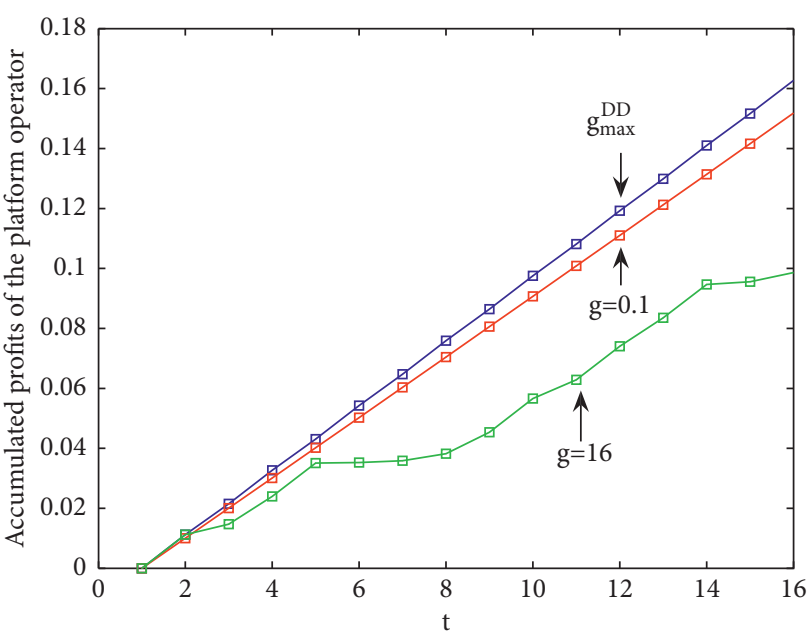

(b)

FIgURe 8: Accumulated profits of the platform operator with different $g$ : (a) $a=1.5, c=0.2, k=0.2$ in system CD and (b) $a=1.5, c=$ $0.2, k=0.2$ in system DD. 
6.1. Who Benefits from the Capacity Sharing? The analysis on whether or not the participants can benefit from the capacity sharing is the premise of the analysis of the choice of trading strategies. When both the capacity requestor and the capacity provider can freely choose whether or not to participate in capacity sharing, they will make a comparison on profits obtained with and without capacity sharing.

Proposition 10. Both the capacity requestor and the capacity provider can benefit from being a seeker in capacity-sharing business; the capacity requestor can also benefit from being a waiter when the potential demand is relatively large, i.e., $a>1$; the capacity provider can also benefit from being a waiter when the production cost is relatively small, i.e., $0<c<\min \{4 a-3,5-4 a\}$.

For the capacity requestor, it can benefit from borrowing capacities from other firms, even from its rival, when it seeks partners proactively. It can also benefit when being a waiter on the condition that the potential demand is relatively large. Hence, if the potential demand is relatively larger, it definitely gets more profits from capacity sharing regardless of the trading styles. For the capacity provider, it will also benefit from being a seeker and sharing capacities with the partners. For the low-cost capacity providers, they can also get more profits by being a waiter. Note that $5-4 a$ is lower than $4 a-3$ when $a>1$. If $a>1$ and $0<c<5-4 a$; both can benefit from capacity sharing regardless of the trading styles. Therefore, apart from the basic condition shown in Proposition 2 and 5; the following conditions should be further met for each trading strategy adoption because a match is successful only if both match-seekers accept the match [11]:

(1) DD: $a>1$

(2) CD: $0<c<\min \{4 a-3,5-4 a\}$

(3) CD and DD: $a>1$ and $0<c<5-4 a$

\subsection{The Choices of Trading Strategies}

Proposition 11. Comparing the profits obtained under CD and DD trading strategies, the following occurs:

(1) The capacity provider: $\pi_{s}^{D D}>\pi_{s}^{C D}$

(2) The capacity $\begin{cases}\pi_{b}^{D D}<\pi_{b}^{C D} & \text { if } \max \left\{0, c_{1}\right\}>c>5-4 a \\ \pi_{b}^{D D}<\pi_{b}^{C D} & \text { otherwise }\end{cases}$ requestor:

(3) The $\begin{cases}\pi_{p}^{D D}>\pi_{p}^{C D} & \text { if } \max \left\{0, c_{2}\right\}<c<5-4 a \\ \pi_{p}^{D D}>\pi_{p}^{C D} & \text { otherwise }\end{cases}$

operator:

Proposition 11 indicates that the capacity provider can always get more profits under DD than the CD trading strategy. It means that the capacity provider can always benefit from seeking capacity requestors for matches, even compared with the extreme case in which only one capacity provider acts as a waiter. Therefore, the capacity provider should actively seek the capacity requestors. For the capacity requestors, the high-cost ones prefer to wait for capacity providers; those low-cost ones prefer to seek capacity providers. A profit-maximizing platform operator should recommend the high-cost firms to accept $C D$ trading strategy and recommend those low-cost firms to accept DD trading strategy.

As the capacity provider will always choose $\mathrm{DD}$, it may be the only all-win choice. Based on Proposition 11, we have the following proposition.

Proposition 12. Note that $c_{2}>c_{1}$, the only all-win situation DD happens on the firms with production cost satisfying $\max \left\{0, c_{1}\right\}<c<c_{2}$.

Theoretically, only the firms with production cost satisfying $\max \left\{0, c_{1}\right\}<c<c_{2}$ can achieve an all-win situation, as shown in Figure 9. Therefore, when the production cost is in a moderate range, the capacity provider, the capacity requestor, and the platform operator can all benefit from a demand-driven trading strategy, that is, the capacity requestor release the demand information, waiting for the capacity providers' quotation.

According to the above analysis, if the capacity requestor's capacity is seriously limited and the production cost is relatively low, the CD trading strategy can be a good option for the capacity requestor to search for the capacity provider. If the capacity requestor's capacity is relatively large and the production cost is not high, the capacity requestor can choose to adopt the DD trading strategy to wait for the capacity provider. When the capacity requestor's capacity is within a medium range and the cost is comparatively moderate, the capacity requestor and the capacity provider can choose between the CD and DD. Under this situation, the capacity requestor with medium capacity and relatively high cost had better choose DD strategy for better profitability, while the capacity provider always gains more profits under the DD trading strategy.

The platform can induce choices of the capacity requestor and the capacity provider by some specific methods. For a profit-maximizing platform operator, it is advisable to suggest the firms with relatively high production costs and with relatively low costs to adopt the CD trading strategy and DD trading strategy, respectively. Therefore, an all-win situation under the DD trading strategy exits for the capacity provider, the capacity requestor, and the platform operator when the production cost is within a certain range. When taking the bounded rationality of the platform operator into account, if the platform operator is bounded rational and highlights the stability of the system, it is wise to recommend the firms with a relatively high cost to accept DD trading strategy. The analysis has shown that the stability performance will be put as the first consideration since the trading strategy with better stability can result in higher long-run profits. Therefore, we propose the decision-making principle for the platform operator to provide insights for its recommendation and analyze the best choice in different situations. 


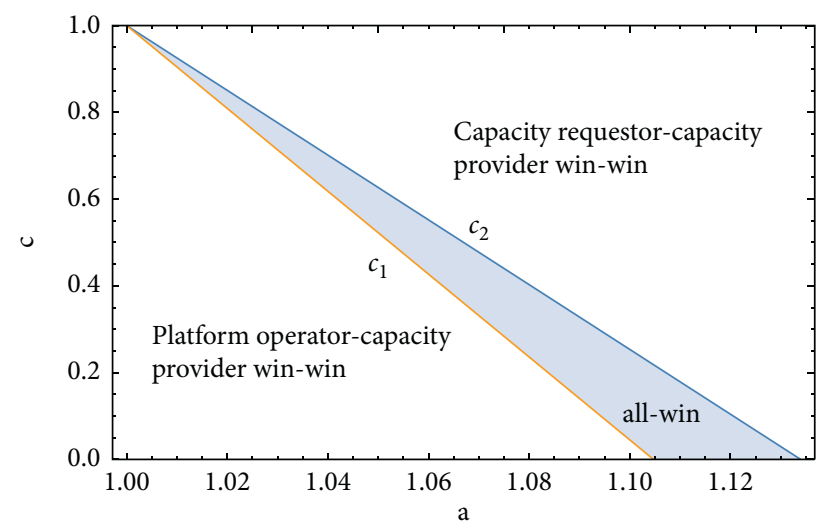

FIGURE 9: Win-win situation.

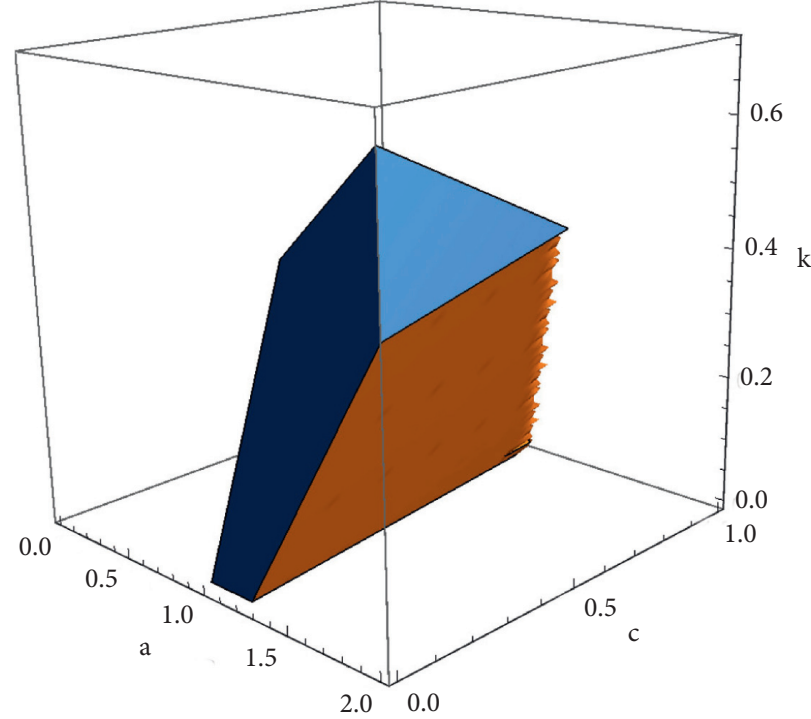

(a)

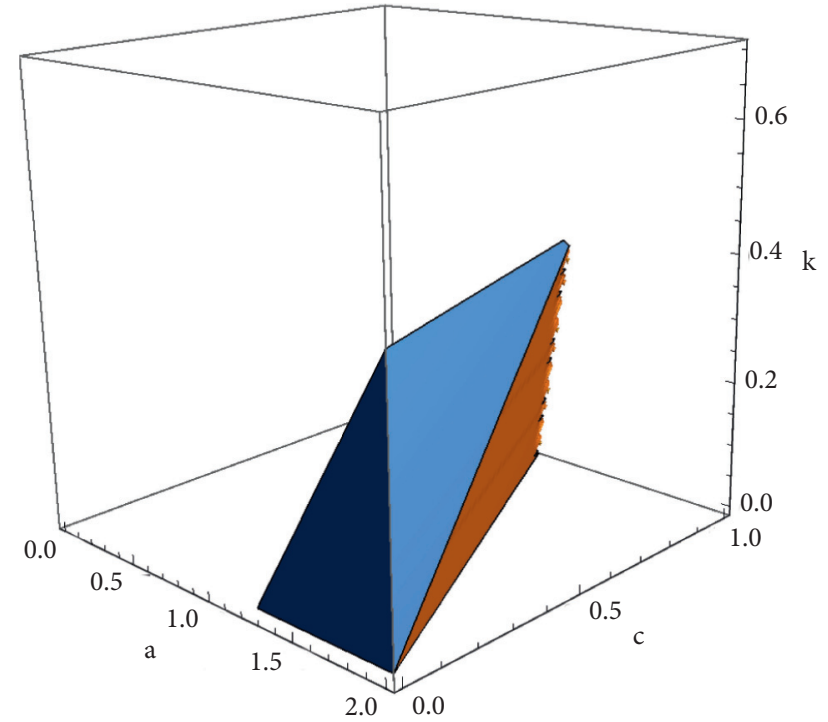

(b)

Figure 10: The existence of both scenarios: (a) the case $c<5+7 k-4 a$ w.r.t. $a, c$, and $k$ and (b) the case $c>5+7 k-4 a$ w.r.t. $a, c$, and $k$.

\section{Conclusions}

In this paper, we model two trading strategies in a capacitysharing supply chain based on the behaviors of the capacity requestor and the capacity provider. We investigate a bounded rational platform operator's preference from these two trading strategies by comparing the profitability performance and stability performance based on the chaos theory. Furthermore, we analyze the preference of the capacity provider and the capacity requestor on the trading strategy and discuss the all-win situation.

The results show that for a profit-maximizing platform operator, it would like to recommend firms with high production costs and firms with low production costs to adopt CD and DD trading strategies, respectively. In contrast, for a bounded rational platform operator, it will obtain better stability by recommending the firms with high production costs to accept the DD trading strategy. Comprehensively, the trading strategy with better stability performance should be put as the first consideration since the trading strategy with better stability can result in higher long-run profits. The choice of trading strategy is influenced by the production cost, the capacity owned by the capacity requestor, and the potential market size. The capacity provider can always obtain higher profits under the DD trading strategy rather than $\mathrm{CD}$. The capacity requestor with high production cost prefers to choose the DD trading strategy, while the one with low production cost prefers to choose the CD strategy. The DD trading strategy will achieve the all-win situation only when the production cost satisfies certain conditions.

In this paper, we investigate the capacity-sharing transaction with considering little distinction in the manufacturing process of the capacity provider and the 
capacity requestor and three members in the capacitysharing supply chain. We will further take the difference in production costs into account and explore the many-tomany relationships of capacity providers and capacity requestors for further research.

\section{Appendix}

\section{A. Exact Expressions of Thresholds}

$$
\begin{aligned}
& k_{1}=-11+6 \sqrt{3}-2(-2+\sqrt{3}) a, \\
& k_{2}=\frac{1}{2}\left(-1+a-\frac{4}{(2+\sqrt{3}) g}\right), \\
& k_{3}=\frac{1}{2}\left(-1+a-\frac{2}{g}\right), \\
& k_{4}=\frac{6-4 \sqrt{3}+(-1+a) g}{2 g}, \\
& k_{5}=1-\frac{8}{g}, \\
& a_{1}=\frac{3 g-8 \sqrt{3}-14}{g}, \\
& a_{2}=3-\frac{4(7+4 \sqrt{3})}{(2+\sqrt{3}) g}, \\
& a_{3}=\frac{-22+4 \sqrt{3}+3 g}{g} .
\end{aligned}
$$

\section{B. Proof of Propositions}

Proof. of Proposition 1.

By backward induction, in stage 3 , the capacity requestor decides on the order quantities from the capacity provider. As $\left(\partial^{2} \pi_{t} / \partial q_{t}^{2}\right)=-2$, the BRF of $q_{t}$ is

$$
q_{t}=\frac{1}{2}\left(-1+2 a-c-3 k-2 q_{s}\right) .
$$

Then we have the BRF of $p_{t}$ is

$$
p_{t}=\frac{1}{2}\left(3-2 a+c+3 k+2 q_{s}\right) .
$$

In stage 2, the capacity provider determines its own production quantity $q_{s}$, which maximizes the capacity provider's profits as $\left(\partial^{2} \pi_{s} / \partial q_{s}^{2}\right)=-2$. The BRF of $q_{s}$ is

$$
q_{s}=\frac{1}{4}\left(-3+4 a-c+2 c_{p}-5 k\right) .
$$

In stage 1 , since $\left(\partial^{2} \pi_{p} / \partial c_{p}^{2}\right)=-1$, a perfect rational platform gets the unique optimal service price $c_{p}^{*}$. Substituting $c_{p}^{*}$ into the BRFs of $q_{t}, p_{t}$, and $q_{s}$, we can get the equilibrium outcomes given in Proposition 1.

In addition, the equilibrium profits are

$$
\begin{aligned}
& \pi_{b}^{\mathrm{CD}}= \frac{1}{64}\left(1+c^{2}+38 k+25 k^{2}-2 c(1+19 k)\right), \\
& \pi_{s}^{\mathrm{CD}}= \frac{1}{64}\left(-15+17 c^{2}-32 a(-1+c-k)-66 k\right. \\
&\left.-47 k^{2}+c(-2+34 k)\right), \\
& \pi_{p}^{\mathrm{CD}}= \frac{1}{32}(-1+c+k)^{2} . \\
& \pi_{b}^{\mathrm{CD}} \text { increases in } k \text { because }\left(\partial^{2} \pi_{b}^{\mathrm{CD}} / \partial k^{2}\right)=(25 / 32) \text { and }
\end{aligned}
$$
the stationary point $k=(19 / 25)(c-1)<0$.

$\pi_{s}^{\mathrm{CD}}$ decreases in $k$ because $\left(\partial^{2} \pi_{s}^{\mathrm{CD}} / \partial k^{2}\right)=-(47 / 32)$ and the stationary point $k=(1 / 47)(16 a+17 c-33)<0$.

Proof. of Proposition 2.

$8 a>5+3 c+11 k$ guarantees $q_{s}^{\mathrm{CD}^{*}}>0 . c+k<1$ guarantees $c_{p}^{\mathrm{CD}^{*}}>0$ and $q_{t}^{\mathrm{CD}}>0$. Note that when $a>1+k, 0<c+$ $k<1$ is sufficient condition for $8 a-3 c>11 k+5$.

Proof. of Proposition 4.

The proof is straightforward by solving the optimization problem (4). In addition, the equilibrium profits are

$$
\begin{aligned}
\pi_{b}^{\mathrm{DD}}= & \frac{1}{12}\left(-4-2 a^{2}+c(2-3 k)-8 k-9 k^{2}\right. \\
& +a(6-2 c+11 k)), \\
\pi_{s}^{\mathrm{DD}}= & \frac{1}{48}\left(4+13 a^{2}+13 c^{2}+4 k+13 k^{2}\right. \\
& -2 a(2+11 c+13 k)+c(-4+22 k)),
\end{aligned}
$$

$$
\pi_{p}^{\mathrm{DD}}=\frac{1}{24}(-2+a+c-k)^{2} .
$$

$\pi_{b}^{\mathrm{DD}}$ increases in $k$ because $\left(\partial^{2} \pi_{b}^{\mathrm{DD}} / \partial k^{2}\right)=-(3 / 2)$ and the stationary point $k=(1 / 18)(-8+11 a-3 c)>a-1$.

$\pi_{s}^{\mathrm{DD}}$ decreases in $k$ because $\left(\partial^{2} \pi_{s}^{\mathrm{DD}} / \partial k^{2}\right)=(13 / 24)$ and the stationary point $k=a+(1 / 13)(-11 c-2)>a-1$.

\section{Proof. of Proposition 5.}

From Proposition 4, we can see that $7 a-5 c-7 k-2>0$ guarantees $q_{s}^{\mathrm{DD}}>0$ and $a+c<2+k$ guarantees $c_{p}^{\mathrm{DD}^{*}}>0$, $q_{t}^{\mathrm{DD}^{*}}>0$, and $p_{t}^{\mathrm{DD}^{*}}>0$. As $a>1+k, a+c<2+k$ is sufficient condition for $7 a-5 c-7 k-2>0$, only when $k>a+c-2$ may both the capacity requestor and the capacity provider participate in the capacity-sharing business with DD trading mechanism. As for the cost $c$, only when $c<2-a+k$ may both participate in the capacity-sharing business with $\mathrm{DD}$ trading mechanism. 
Proof. of Proposition 7.

$$
\pi_{p}^{\mathrm{CD}}-\pi_{p}^{\mathrm{DD}}=\frac{1}{96}\left(-4(-2+a+c-k)^{2}+3(-1+c+k)^{2}\right) .
$$

$$
\begin{aligned}
& \text { We can get } \pi_{p}^{\mathrm{CD}}>\pi_{p}^{\mathrm{DD}} \text { when } \\
& c<c_{\text {th }}^{\pi_{p}}=((-4+\sqrt{3}+2 a-(2+\sqrt{3}) k) /(-2+\sqrt{3})) \text {, and } \\
& \text { we can get } \pi_{p}^{\mathrm{CD}}<\pi_{p}^{\mathrm{DD}} \text { when } \\
& c>c_{\mathrm{th}}^{\pi_{p}}=((-4+\sqrt{3}+2 a-(2+\sqrt{3}) k) /(-2+\sqrt{3})) \text {. }
\end{aligned}
$$

$$
\begin{gathered}
\frac{-4+\sqrt{3}+2 a-(2+\sqrt{3}) k}{-2+\sqrt{3}}-(1-k)=\frac{2(-1+a-2 k)}{-2+\sqrt{3}}, \\
\frac{-4+\sqrt{3}+2 a-(2+\sqrt{3}) k}{-2+\sqrt{3}}-(2+k-a)=\frac{\sqrt{3}(-1+a-2 k)}{-2+\sqrt{3}} .
\end{gathered}
$$

If $-1+a-2 k>0$, we have $((-4+\sqrt{3}+2 a-(2+\sqrt{3}) k)$ $/(-2+\sqrt{3}))<\min \{1-k, 2+k-a\}=c_{\max }$.

Proof. of Proposition 8.

Since

$g_{\max }-g_{\max }=(2(-5+4 a+c-7 k) /(-2+a+c-$

k) $(-1+c+k))$ and $-2+a+c-k<0$, and $-1+c+k<0$, we can get when $-5+4 a+c-7 k>0, g_{\max ^{D D}}-g_{\max }{ }^{C D}>0$, and when $-5+4 a+c-7 k<0, g_{\max ^{\mathrm{DD}}}-g_{\max }<0$. The threshold is $c_{\text {th }}^{g_{\max }}=5+7 k-4 a$.

To prove the existence of both scenarios, we draw Figure 10:

Proof. of Proposition 9.

The optimal decision changes with respect to $c$, which determines not only the stability performance but also profitability performance. Take scenario 1 for example. Table 2 gives the orders of the thresholds of $c$, that is, $0<c_{\text {th }}^{\pi_{p}}<c_{\min ^{\mathrm{DD}}}<c_{\min ^{\mathrm{CD}}}<c_{\max }$.

(1) When $c$ locates in the range $\left(0, c_{\mathrm{th}}^{\pi_{p}}\right)$ or $\left(c_{\mathrm{th}}^{\pi_{p}}, c_{\min ^{\mathrm{DD}}}\right)$, both system CD and DD are unstable; then none of the strategies is optimal.

(2) When $c$ locates in the range $\left(c_{\min ^{\mathrm{DD}}}, c_{\min ^{\mathrm{CD}}}\right)$, the system DD is stable, but $\mathrm{CD}$ is unstable. Then the only stable DD is selected, although the profit under $\mathrm{DD}$ is lower than that under $\mathrm{CD}$ (i.e., submaximum), as $\pi_{p}^{\mathrm{DD}}<\pi_{p}^{\mathrm{CD}}$ when $c>c_{\text {th }}$.

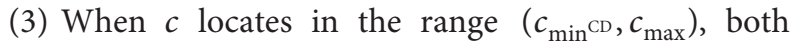
system DD and CD are stable. Then CD is selected as $\pi_{p}^{\mathrm{DD}}<\pi_{p}^{\mathrm{CD}}$ when $c>c_{\text {th }}^{\pi_{p}}$.

In scenarios 2,3 , and 4 , the optimal decisions are obtained in the same way; here, we omit the proof process.
Proof. of Proposition 10.

$$
\begin{aligned}
& \pi_{b}^{\mathrm{DD}}-\pi_{b}^{\mathrm{no}}=-\frac{1}{6}(-1+a)(-2+a+c), \\
& \pi_{s}^{\mathrm{DD}}-\pi_{s}^{\mathrm{no}}=\frac{1}{48}(-2+a+c)^{2}, \\
& \pi_{p}^{\mathrm{DD}}-\pi_{p}^{\mathrm{no}}=\frac{1}{24}(-2+a+c)^{2}, \\
& \pi_{b}^{\mathrm{CD}}-\pi_{b}^{\mathrm{no}}=\frac{1}{64}(-1+c)^{2}, \\
& \pi_{s}^{\mathrm{CD}}-\pi_{s}^{\mathrm{no}}=\frac{1}{64}\left(-15+32 a-16 a^{2}-2 c+c^{2}\right), \\
& \pi_{p}^{\mathrm{CD}}-\pi_{p}^{\mathrm{no}}=\frac{1}{32}(-1+c)^{2} .
\end{aligned}
$$

Facing DD, the capacity provider and platform always benefit from capacity sharing under DD; the capacity requestor can benefit when $a>1$.

Facing CD, the capacity requestor and platform always benefit from capacity sharing under $\mathrm{CD}$; the capacity provider can benefit when $(1 / 4)(3+c)<a<(1 / 4)(5-c)$ or $0<c<\min \{4 a-3,5-4 a\}$. If $a>1$, we have $0<c<5-4 a$ benefit, $5-4 a<c<1$ lose. If $a<1$, we have $0<c<4 a-3$ benefit and $4 a-3<c<1$ lose.

We can see that there is always one between $4 a-3$ and $5-4 a$, which is lower than 1 . Therefore, the upper bound of $c$ will be $4 a-3$ or $5-4 a$, instead of the basic condition in Proposition 2. If $a>1$, we have $5-4 a<4 a-3$, and the upper bound is $5-4 a$. Similarly, if $a<1$, we have $5-4 a>4 a-3$, and the upper bound is $4 a-3$. 
In $\mathrm{CD}$ and $\mathrm{DD}$ case, the upper bound $5-4 a$ also meets the basic condition $c<\min \{1,2-a\}$ shown in Propositions 2 and 5 with $k=0$.
Proof. of Proposition 11.

$$
\begin{aligned}
& \pi_{b}^{\mathrm{DD}}-\pi_{b}^{\mathrm{CD}}=\frac{1}{192}\left(-67-32 a^{2}-32 a(-3+c)+38 c-3 c^{2}\right) \\
& \pi_{s}^{\mathrm{DD}}-\pi_{s}^{\mathrm{CD}}=\frac{1}{192}\left(61+52 a^{2}+8 a(-14+c)-10 c+c^{2}\right) \\
& \pi_{p}^{\mathrm{DD}}-\pi_{p}^{\mathrm{CD}}=\frac{1}{96}\left(-3(-1+c)^{2}+4(-2+a+c)^{2}\right) .
\end{aligned}
$$

Note that $c_{1}=(1 / 3)(19-4 \sqrt{10}(a-1)-16 a)$ and $c_{2}=5-2 \sqrt{3}(a-1)-4 a$; it is not difficult to prove $c_{1}<c_{2}<5-4 a$ when $a>1$, then we have:

$$
\begin{aligned}
& \begin{cases}\pi_{p}^{\mathrm{DD}}<\pi_{p}^{\mathrm{CD}}, & \text { if } \max \left\{0, c_{2}\right\}<c<5-4 a, \\
\pi_{p}^{\mathrm{DD}}>\pi_{p}^{\mathrm{CD}}, & \text { otherwise, } a<\frac{4-\sqrt{3}}{2},\end{cases} \\
& \begin{cases}\pi_{s}^{\mathrm{DD}}<\pi_{s}^{\mathrm{CD}}, & \text { false, } \\
\pi_{s}^{\mathrm{DD}}>\pi_{s}^{\mathrm{CD}}, & \text { always true, }\end{cases} \\
& \begin{cases}\pi_{b}^{\mathrm{DD}}>\pi_{b}^{\mathrm{CD}}, & \text { if max }\left\{0, c_{1}\right\}<c<5-4 a, \\
\pi_{b}^{\mathrm{DD}}<\pi_{b}^{\mathrm{CD}}, & \text { otherwise, } a<\frac{12-\sqrt{10}}{8} .\end{cases}
\end{aligned}
$$

Proof. of Proposition 12.

Using subtraction, we can get the following result.

$$
c_{2}-c_{1}=-\frac{2}{3}(-2+3 \sqrt{3}-2 \sqrt{10})(-1+a)>0 \text {. }
$$

\section{Data Availability}

The data used to support the findings of our paper have been included within the article.

\section{Conflicts of Interest}

The authors declare that there are no conflicts of interest.

\section{Acknowledgments}

This research was supported by the Fundamental Research Funds of Qilu University of Technology (Shandong Academy of Sciences) and the Humanities and Social Science Research and Innovation Team of Digital Transformation and Innovation Management of Qilu University of Technology (Shandong Academy of Sciences).

\section{References}

[1] G. Duménil and D. Lévy, "Why does profitability matter? Profitability and stability in the U.S. Economy since the 1950s," Review of Radical Political Economics, vol. 25, no. 1, pp. 27-61, 1993.

[2] K. J. Euske and A. Riccaboni, "Stability to profitability: managing interdependencies to meet a new environment," Accounting, Organizations and Society, vol. 24, no. 5-6, pp. 463-481, 1999.

[3] L. Guo and X. Wu, "Capacity sharing between competitors," Management Science, vol. 64, pp. 3554-3573, 2017.

[4] P. Renna and P. Argoneto, "Capacity sharing in a network of independent factories: a cooperative game theory approach," Robotics and Computer-Integrated Manufacturing, vol. 27, no. 2, pp. 405-417, 2011.

[5] S.-J. S. Yang and E. J. Anderson, "Competition through capacity investment under asymmetric existing capacities and costs," European Journal of Operational Research, vol. 237, no. 1, pp. 217-230, 2014.

[6] H. Seok and S. Y. Nof, "Dynamic coalition reformation for adaptive demand and capacity sharing," International Journal of Production Economics, vol. 147, pp. 136-146, 2014. 
[7] G. Wang, G. Zhang, X. Guo, and Y. Zhang, "Digital twindriven service model and optimal allocation of manufacturing resources in shared manufacturing," Journal of Manufacturing Systems, vol. 59, pp. 165-179, 2021.

[8] H. Yang, R. Chen, and S. Kumara, "Stable matching of customers and manufacturers for sharing economy of additive manufacturing," Journal of Manufacturing Systems, vol. 61, pp. 288-299, 2021.

[9] P. Li and P. Jiang, "Enhanced agents in shared factory: enabling high-efficiency self-organization and sustainability of the shared manufacturing resources," Journal of Cleaner Production, vol. 292, Article ID 126020, 2021.

[10] J. Qin, K. Wang, Z. Wang, and L. Xia, "Revenue sharing contracts for horizontal capacity sharing under competition," Annals of Operations Research, vol. 291, pp. 1-30, 2018.

[11] A. Basu, S. Bhaskaran, and R. Mukherjee, "An analysis of search and authentication strategies for online matching platforms," Management Science, vol. 65, pp. 2412-2431, 2019.

[12] D. Zhao, H. Han, J. Shang, and J. Hao, "Decisions and coordination in a capacity sharing supply chain under fixed and quality-based transaction fee strategies," Computers \& Industrial Engineering, vol. 150, Article ID 106841, 2020.

[13] F. Ye, D. Ni, and K. W. Li, "Competition between manufacturers and sharing economy platforms: an owner base and sharing utility perspective," International Journal of Production Economics, vol. 234, Article ID 108022, 2021.

[14] Á. Szaller, G. Pedone, P. Egri, Á. Szalóki, and G. Nick, “A mutualistic framework for sustainable capacity sharing in manufacturing," Procedia CIRP, vol. 93, pp. 938-943, 2020.

[15] M. Li, Z. Li, X. Huang, and T. Qu, "Blockchain-based digital twin sharing platform for reconfigurable socialized manufacturing resource integration," International Journal of Production Economics, vol. 240, Article ID 108223, 2021.

[16] A. Surana, S. Kumara, M. Greaves, and U. N. Raghavan, "Supply-chain networks: a complex adaptive systems perspective," International Journal of Production Research, vol. 43, no. 20, pp. 4235-4265, 2005.

[17] U. Jüttner, H. Peck, and M. Christopher, "Supply chain risk management: outlining an agenda for future research," International Journal of Logistics Research and Applications, vol. 6, no. 4, pp. 197-210, 2003.

[18] Z. Lei, Y. j. Li, and Y. q. Xu, "Chaos synchronization of bullwhip effect in a supply chain," in Proceedings of the 2006 International Conference on Management Science and Engineering, pp. 557-560, IEEE, Lille, France, October 2006.

[19] J. Ma, W. Lou, and Y. Tian, "Bullwhip effect and complexity analysis in a multi-channel supply chain considering price game with discount sensitivity," International Journal of Production Research, vol. 57, no. 17, pp. 5432-5452, 2019.

[20] R. D. Wilding, "Chaos theory: implications for supply chain management," The International Journal of Logistics Management, vol. 9, no. 1, pp. 43-56, 1998.

[21] D. Stapleton, J. B. Hanna, and J. R. Ross, "Enhancing supply chain solutions with the application of chaos theory," Supply Chain Management: An International Journal, vol. 11, no. 2, pp. 108-114, 2006.

[22] H. B. Hwarng and N. Xie, "Understanding supply chain dynamics: a chaos perspective," European Journal of Operational Research, vol. 184, no. 3, pp. 1163-1178, 2008.

[23] H. B. Hwarng and X. Yuan, "Interpreting supply chain dynamics: a quasi-chaos perspective," European Journal of Operational Research, vol. 233, no. 3, pp. 566-579, 2014.

[24] X. Zhan, J. Ma, Y. Li, and L. Zhu, "Design and coordination for multi-channel recycling of oligopoly under the carbon tax mechanism," Journal of Cleaner Production, vol. 223, pp. 413-423, 2019.

[25] J. Ma and L. Xie, "The comparison and complex analysis on dual-channel supply chain under different channel power structures and uncertain demand," Nonlinear Dynamics, vol. 83, no. 3, pp. 1379-1393, 2016.

[26] F. Zhang and C. Wang, "Dynamic pricing strategy and coordination in a dual-channel supply chain considering service value," Applied Mathematical Modelling, vol. 54, pp. 722-742, 2018.

[27] H. Tu, X. Mao, and X. Wang, "Complexity of a dynamic hybrid supply chain game model with a service factor," Nonlinear Dynamics, vol. 97, no. 4, pp. 2055-2066, 2019.

[28] J. Ma, L. Zhu, and Y. Guo, "Strategies and stability study for a triopoly game considering product recovery based on closedloop supply chain," Operational Research, vol. 21, no. 4, pp. 1-22, 2019.

[29] L. Xie and J. Ma, "Study the complexity and control of the recycling-supply chain of China's color TVs market based on the government subsidy," Communications in Nonlinear Science and Numerical Simulation, vol. 38, pp. 102-116, 2016.

[30] A. Göksu, U. E. Kocamaz, and Y. Uyaroğlu, "Synchronization and control of chaos in supply chain management," Computers \& Industrial Engineering, vol. 86, pp. 107-115, 2015.

[31] U. E. Kocamaz, H. Taşkın, Y. Uyaroğlu, and A. Göksu, "Control and synchronization of chaotic supply chains using intelligent approaches," Computers \& Industrial Engineering, vol. 102, pp. 476-487, 2016.

[32] A. Dixit, "Comparative statics for oligopoly," International Economic Review, vol. 27, no. 1, pp. 107-122, 1986.

[33] G. I. Bischi, M. Gallegati, and A. Naimzada, "Symmetrybreaking bifurcations and representative firm in dynamic duopoly games," Annals of Operations Research, vol. 89, pp. 252-271, 1999.

[34] E. Jury, "A simplified stability criterion for linear discrete systems," Proceedings of the Ire, vol. 50, no. 6, pp. 1493-1500, 1962. 